\title{
Altered ISGylation drives aberrant macrophage-dependent immune responses during SARS-CoV-2 infection
}

\author{
Deeksha Munnur ${ }^{1,13}$, Qiwen Teo ${ }^{2,13}$, Denzel Eggermont $\mathbb{B}^{3,4,13}$, Horace H. Y. Lee $\mathbb{1}^{2,5,13}$, \\ Fabien Thery $\mathbb{1}^{3}$, Julian $\mathrm{Ho}^{2}$, Sophie Wilhelmina van Leur ${ }^{1}$, Wilson W. S. Ng$^{2}$, Lewis Y. L. Siu' \\ Antje Beling ${ }^{6,7}$, Hidde Ploegh ${ }^{8}$, Adan Pinto-Fernandez ${ }^{9}$, Andreas Damianou ${ }^{9}$, Benedikt Kessler ${ }^{9}{ }^{9}$, \\ Francis Impens $s^{3,4,10 凶}$, Chris Ka Pun Mok $\mathbb{D}^{2,11,12 \bowtie}$ and Sumana Sanyal ${ }^{1,2} \bowtie$
}

\begin{abstract}
Ubiquitin-like protein ISG15 (interferon-stimulated gene 15) (ISG15) is a ubiquitin-like modifier induced during infections and involved in host defense mechanisms. Not surprisingly, many viruses encode delSGylating activities to antagonize its effect. Here we show that infection by Zika, SARS-CoV-2 and influenza viruses induce ISG15-modifying enzymes. While influenza and Zika viruses induce ISGylation, SARS-CoV-2 triggers delSGylation instead to generate free ISG15. The ratio of free versus conjugated ISG15 driven by the papain-like protease (PLpro) enzyme of SARS-CoV-2 correlates with macrophage polarization toward a pro-inflammatory phenotype and attenuated antigen presentation. In vitro characterization of purified wild-type and mutant PLpro revealed its strong delSGylating over deubiquitylating activity. Quantitative proteomic analyses of PLpro substrates and secretome from SARS-CoV-2-infected macrophages revealed several glycolytic enzymes previously implicated in the expression of inflammatory genes and pro-inflammatory cytokines, respectively. Collectively, our results indicate that altered free versus conjugated ISG15 dysregulates macrophage responses and probably contributes to the cytokine storms triggered by SARS-CoV-2.
\end{abstract}

\begin{abstract}
nterferons (IFNs) are the first line of defense against virus infections $^{1}$ and are critical drivers of the innate immune response. Although hosts deficient in type I IFN are more susceptible to virus infections ${ }^{2}$, an excess of type I IFN or aberrant cytokine response may lead to extensive tissue damage as is commonly observed in highly pathogenic cases of influenza ${ }^{3}$ and coronaviruses $^{4}$. Mice lacking the IFN- $\alpha$ receptor (IFNAR) have a higher survival rate to influenza and coronavirus infections than wild-type (WT) animals ${ }^{5,6}$, again pointing to a dysregulation of IFN signaling underpinning the immunopathology of severe cases.

Macrophages are cells of the innate immune system that play key roles in modulating disease severity during virus infections. They can be infected by a range of viruses and are the major producers of pro-inflammatory cytokines, such as tumor necrosis factor- $\alpha$ (TNF- $\alpha$ ), IFN- $\beta$, IP-10 (C-X-C motif chemokine 10) and monocyte chemoattractant protein 1 (MCP-1), which have an impact on the pathogenesis and clinical outcomes in the host ${ }^{7-10}$. Regulation of cytokines in macrophages is essential. Overproduction of cytokines, commonly referred to as 'cytokine storms', aggravates lung damage with uncontrolled extravasation of immune cells into infection sites ${ }^{4,11}$, although the exact sequence of events is not yet completely understood.
\end{abstract}

ISG15 is a ubiquitin-like modifier with broad-spectrum antiviral activity that is part of the first line of defense against pathogens. Post-translational modifications by ubiquitin and ISG15 are frequently targeted by viruses to perturb host immune responses ${ }^{12}$. ISG15 can be conjugated to proteins in a process termed ISGylation or be secreted in its free form. Among the hundreds of modifiable substrates, many have immune-related functions ${ }^{13-15}$, and ISG15 (free or conjugated) has been shown to protect the host against infections ${ }^{16}$.

The fate of ISGylation in virus-infected macrophages has not been reported so far. The impact of ISG15 has only been investigated in influenza virus-infected epithelial cells where ISG15-modified influenza NS1 inhibited virus replication. In addition, ISGylated TSG101, a member of the endosomal sorting complexes required for transport, inhibited transport of influenza virus proteins ${ }^{15,17}$. However, much of the immune response at the site of infection emanates from monocyte-derived and tissue-resident macrophages. In this study, we investigated how viruses interact with the immune activation pathways in infected macrophages using Zika and SARS-CoV-2 as two (+)RNA viruses from distinct families. Zika is a member of the Flaviviridae family while SARS-CoV-2 is

\footnotetext{
'Sir William Dunn School of Pathology, South Parks Road, University of Oxford, Oxford, UK. '2HKU-Pasteur Research Pole, School of Public Health, Li Ka Shing Faculty of Medicine, University of Hong Kong, Hong Kong SAR, Hong Kong. ${ }^{3}$ VIB-UGent Center for Medical Biotechnology, Ghent, Belgium. ${ }^{4}$ Department of Biomolecular Medicine, Ghent University, Ghent, Belgium. ${ }^{5}$ Department of Pathology, University of Hong Kong, Hong Kong SAR, Hong Kong. ${ }^{6}$ Charité-Universitätsmedizin Berlin, corporate member of Freie Universität Berlin and Humboldt-Universität zu Berlin, Institute of Biochemistry, Berlin, Germany. ${ }^{7}$ Deutsches Zentrum für Herz-Kreislauf-Forschung, partner site Berlin, Germany. ${ }^{8}$ Boston Children's Hospital and Harvard Medical School, Boston, MA, USA. ${ }^{9}$ Mass Spectrometry Laboratory, Target Discovery Institute, Nuffield Department of Medicine, University of Oxford, Oxford, UK. ${ }^{10} \mathrm{VIB}$ Proteomics Core, Ghent, Belgium. "Li Ka Shing Institute of Health Sciences, Faculty of Medicine, The Chinese University of Hong Kong, Hong Kong SAR, Hong Kong. ${ }^{12}$ The Jockey Club School of Public Health and Primary Care, Faculty of Medicine, The Chinese University of Hong Kong, Hong Kong SAR, Hong Kong. ${ }^{13}$ These authors contributed equally: Deeksha Munnur, Qiwen Teo, Denzel Eggermont, Horace H. Y. Lee.

凶e-mail: francis.impens@vib-ugent.be; kapunmok@cuhk.edu.hk; sumana.sanyal@path.ox.ac.uk
} 
a coronavirus. Both Zika and SARS-CoV-2 are single-stranded, positive-sense RNA viruses that replicate in the cytoplasm within membrane-delineated replication organelles. Macrophages are permissive to infection by both viruses; however, they are the primary target cells of Zika virus and support replication to high titers. We also compared these responses to those infected with influenza virus, which is an orthomyxovirus with a vastly different genome organization (negative-sense segmented RNA) and unlike the former, replicates in the nucleus.

Influenza and Zika viruses promoted cellular ISGylation while SARS-CoV-2 triggered secretion of ISG15. Expression of the WT but not the catalytically inactive SARS-CoV-2 PLpro alone was sufficient to drive deISGylation and aberrant macrophage responses. Proteomic analyses revealed that glycolytic enzymes that regulate inflammatory responses are the primary substrates of PLpro deISGylase activity. The secretome from SARS-CoV-2-infected macrophages also revealed enrichment of nonclassical secretory components and pro-inflammatory cytokines. Collectively, our data underscore the critical impact of altered free versus conjugated ISG15 on macrophage function, potentially underpinning the onset of lymphopenia and cytokine storms during infections by SARS-CoV-2.

\section{Results}

Zika and SARS-CoV-2 induce expression of ISGylation enzymes. ISG15 is produced during virus infections downstream of type I IFNs $^{18,19}$. Upregulation of ISG15 and modifying enzymes, including the 1 activating enzyme ubiquitin-like modifier-activating enzyme 7 (UBE1L), E2 conjugation enzyme ubiquitin/ISG15-conjugating enzyme E2 L6 (UBE2L6/UBCH8), E3 ISG15-protein ligase HERC5 (HERC5) and deISGylase Ubl carboxyl-terminal hydrolase 18 (USP18) have been reported, albeit only in virus-infected epithelial cells $^{20,21}$. To determine the magnitude of expression of ISG15 and its modifying enzymes in virus-infected macrophages, we performed quantitative PCR with reverse transcription (RT-qPCR) to quantify the messenger RNA levels of ISG15, UBE1L (E1), UBE2L6/ UBCH8 (E2), HERC5 (E3) and USP18 on Zika and SARS-CoV-2 infection. We treated macrophages with IFN- $\beta$ as positive control, where ISG15 and its conjugating enzymes were all induced as anticipated (Fig. 1a-d). In those infected by either Zika or SARS-CoV-2, all enzymes of the ISGylation pathway were substantially induced (Fig. 1a-e). We also measured their expression in influenza A-infected cells. Like +RNA viruses, in cells infected by human influenza $A(\mathrm{H} 1 \mathrm{~N} 1)$ or avian influenza $A(\mathrm{H} 9 \mathrm{~N} 2)$ virus, expression of all mRNAs, with the exception of UBE1L, was induced to similar levels (Supplementary Fig. 1a-e). Intracellular ISG15 protein, measured by ELISA, was equivalently upregulated after infection with influenza (Supplementary Fig. 1f), Zika virus and most significantly SARS-CoV-2 (Fig. 1f). These results indicate that ISG15 and modifying enzymes are expressed in macrophages and markedly induced after virus infection.

Cellular ISGylation was induced in Zika-infected macrophages (Fig. 1g). Interestingly, although the conjugating enzymes were induced on SARS-CoV-2 infection, total protein ISGylation was markedly low (Fig. 1g), which suggests that SARS-CoV-2 can probably trigger deISGylation of cellular substrates. In influenza-infected macrophages, bulk ISGylation in cell lysates was detectable for both avian (H9N2/Y280), mammalian-adapted (H9N2/Y280-PB2-627K) or pandemic (H1N1/CA04) influenza strains (Supplementary Fig. 1g).

Although type I IFN signaling is a well-known pathway that induces ISG15 and ISGylating enzymes ${ }^{15}$, type I IFN-independent mechanisms have also been proposed ${ }^{22,23}$. To investigate whether virus infection-induced ISG15 expression was dependent on type I IFN signaling, we infected macrophages in the presence of neutralizing antibodies directed against type I IFN receptor (anti-IFN- $\alpha / \beta$ receptor 2) or isotypic control. ISG15 expression was determined by RT-qPCR (Supplementary Fig. 1h,i) and western blotting (Supplementary Fig. 1j). In the presence of anti-IFNAR, ISG15 mRNA expression and free and conjugated ISG15 was diminished compared to isotypic control. Collectively, these data indicate that in virus-infected macrophages, IFN-dependent signaling triggered the expression and conjugation of ISG15, which in turn are hydrolyzed effectively by SARS-CoV-2.

Free ISG15 is secreted via unconventional mechanisms. Apart from its function as a Ubl protein modifier, ISG15 is also known to function as a free, nonconjugated $\operatorname{protein}^{24}$, which can be secreted into the extracellular space ${ }^{25}$. Mice harboring a deletion of UBE1L (E1), which abolishes ISGylation, has been reported to survive better than $I s g 15^{-1-}$, which abolishes both free and conjugated ISG15, suggesting that both the free and conjugated forms of ISG15 may play protective roles in virus infections ${ }^{16}$. We detected extracellular ISG15 after infection by Zika, SARS-CoV-2 and influenza viruses but not from cells stimulated by type I IFN alone or by infection with ultraviolet (UV)-inactivated viruses (Fig. 2a,b). The highest amounts of secreted ISG15 were detected from SARS-CoV-2-infected cells (Fig. 2a). By contrast, infection with UV-inactivated Zika or SARS-CoV-2 did not trigger secretion of ISG15 at all, indicating that live virus is necessary for this process (Fig. 2b). Although a comparable extent of ISG15 mRNA induction was detected in normal human bronchial epithelial (NHBE) cells, dendritic cells (DCs) and macrophages (Supplementary Fig. 3a,b), secretion of ISG15 was detectable only from DCs and macrophages, with the highest amounts from the latter, in particular from those infected with SARS-CoV-2 (Supplementary Fig. 3c-e). These results indicate that respiratory viruses, such as influenza and coronaviruses, both of which have the potential to cause 'cytokine storms' trigger secretion of ISG15 from immune cells to a significantly greater extent compared to Zika virus, which is well adapted to replicate in macrophages and therefore likely able to circumvent macrophage-mediated immune responses.

The process of ISG15 secretion is not well characterized and has been proposed to occur from granulocytes via unconventional mechanisms ${ }^{26,27}$. To exclude the possibility that the extracellular pool of ISG15 was due to cell lysis, apoptosis or via the conventional secretory pathway (Supplementary Fig. 2a), we measured lactate dehydrogenase (LDH) released from infected cells (Fig. 2c and Supplementary Fig. 2e). We also measured extracellular ISG15 secretion on treatment with either caspase inhibitors (Fig. 2d and Supplementary Fig. 2f) or Brefeldin A to block the conventional secretory pathway (Fig. 2e and Supplementary Fig. 2c). Neither of these treatments inhibited ISG15 secretion. Brefeldin A did not affect ISG15 secretion from cells infected with either Zika, SARS-CoV-2 or influenza virus (Fig. 2e and Supplementary Fig. 2c), nor did it appreciably alter intracellular levels of ISG15 (Supplementary Fig. 2d) in infected cells, indicating that it is probably secreted via unconventional mechanisms.

Different mechanisms of unconventional secretory pathways have been reported previously. Among these are a recently identified TMED10-dependent secretory pathway ${ }^{28}$, LC3-dependent extracellular vesicle loading and secretion (LDELS) pathway ${ }^{29}$, secretory autophagosomes ${ }^{30}$ and lysosomes ${ }^{31}$ (Fig. 2f). Depletion of TMED10 did not appreciably alter ISG15 secretion from SARS-CoV-2-infected macrophages (Fig. 2g). The key components of the LDELS pathway are LC3 and neutral sphingomyelinase 2 (nSMase2) $\left(\right.$ ref. $^{29}$ ). Depletion of LC3 abolished secreted ISG15; however, loss of nSMase2 did not, suggesting that LC3 might regulate ISG15 secretion via a different process (Fig. 2h). Secretory lysosomes have been proposed to be critical for egress of coronaviruses. However, inhibiting key components of the secretory lysosomal pathway also did not affect ISG15 secretion (Fig. 2i). Instead, loss of secretory autophagosomal ${ }^{30}$ factors abolished ISG15 secretion 

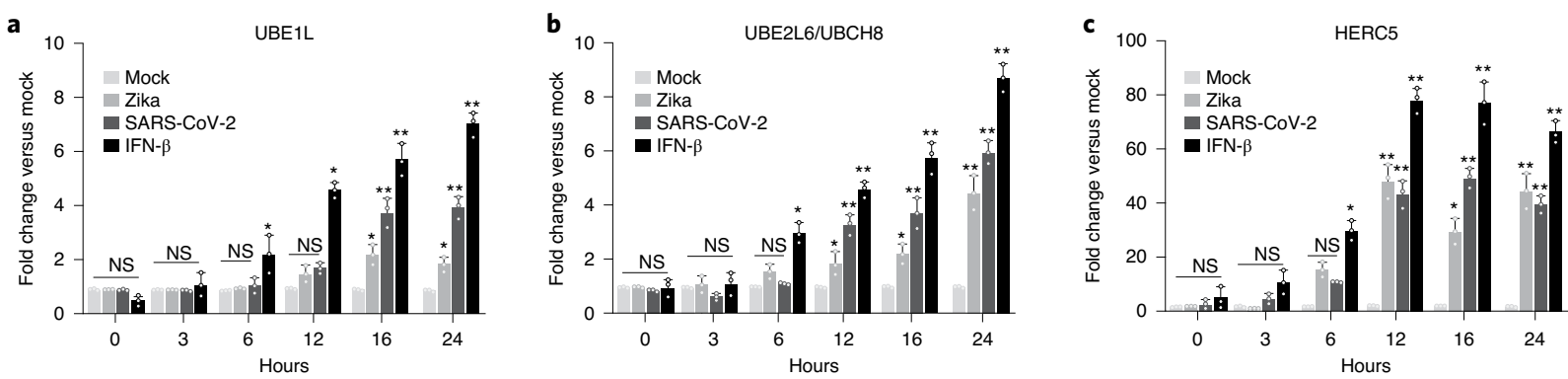

d

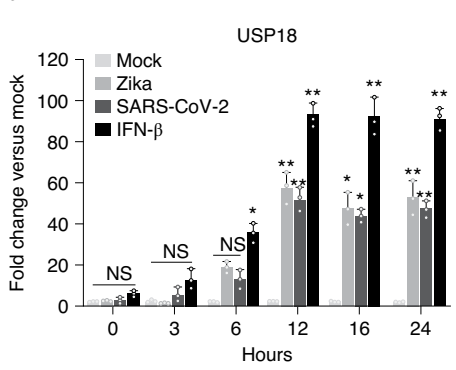

e

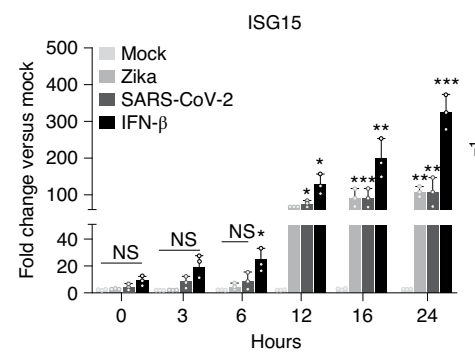

f

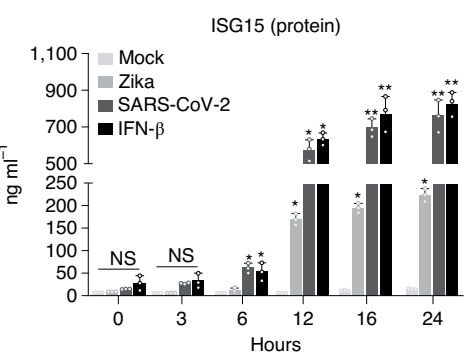

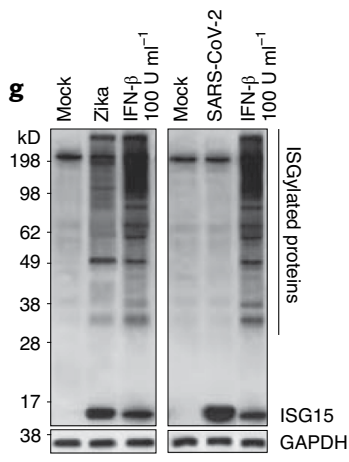

Fig. 1 | The ISGylation machinery is induced in Zika and SARS-CoV-2 virus-infected macrophages. a-e, Macrophages were infected with Zika or SARS-CoV-2 at an MOI of 2. At the indicated time intervals, changes in mRNA expression levels of the ISG15-modifying enzymes UBE1L (a), UBE2L6/UBCH8 (b), HERC5 (c) and USP18 (d), and ISG15 (e) against mock infection were quantified by qPCR. NS, not significant. f, Intracellular ISG15 protein levels in Zika- or SARS-CoV-2-infected macrophages were quantified by ELISA. a-f, Data are displayed as the means \pm s.e.m. of three biologically independent experiments. ${ }^{\star} P<0.05,{ }^{\star \star} P<0.01,{ }^{\star \star \star} P<0.001$ by two-tailed Mann-Whitney $U$-test versus mock-infected cells; $n=3$. g, ISGylation in virus-infected macrophages was measured for Zika and SARS-CoV-2. Macrophages were infected with Zika (left) and SARS-CoV-2 (right) at an MOI of 2. Lysates were collected in 50-mM HEPES supplemented with 0.5\% IGEPAL, pH 7.4, separated by SDS-polyacrylamide gel electrophoresis (SDS-PAGE) and visualized by western blotting using an anti-human ISG15. Glyceraldehyde-3-phosphate dehydrogenase (GAPDH) levels were measured as the loading control. The blot is representative of results obtained from three independent experiments.

(Fig. $2 \mathrm{j}, \mathrm{k}$ ). To characterize this pathway in further detail, we systematically depleted known components of the secretory autophagy pathway $^{32}$. Depletion of the ULK1 complex of the early autophagy pathway, Lyn kinase, which we recently showed to function in this pathway $^{32}$, STX3/STX4, the SNARE complex of secretory autophagy but not STX17, which functions in degradative autophagy blocked ISG15 secretion. Collectively, these data indicate that SARS-CoV-2 infection can specifically trigger secretion of free ISG15 via autophagy-dependent unconventional mechanisms, with the highest amounts detected from SARS-CoV-2-infected macrophages.

SARS-CoV-2 infection triggers aberrant immune responses. The dramatically reduced ISGylation (Fig. 1) and increase in secreted ISG15 (Fig. 2) from SARS-CoV-2-infected macrophages prompted us to investigate the general macrophage immune responses in SARS-CoV-2 infection. Clinical samples from patients with COVID-19 have already indicated aberrant early immune responses in SARS-CoV-2 infection, often accompanied by lymphopenia and secretion of pro-inflammatory cytokines ${ }^{33,34}$. To measure macrophage effector functions, we infected cells with SARS-CoV-2 at a multiplicity of infection (MOI) of 2. At $24 \mathrm{~h}$ post-infection, surface presentation of both major histocompatibility complex class I (MHC-I) and class II (MHC-II) were significantly downregulated specifically in the virus-infected population but not with UV-inactivated virus control or with double-stranded RNA (dsRNA) transfection, a phenomenon that was also evident in Zika-infected cells (Fig. 3a,b and Supplementary Fig. 4a,b). The effect on MHC in influenza-infected cells was far more modest and in accordance with previous reports, which showed downregulation of MHC-I in influenza B-infected cells compared to influenza
$\mathrm{A}^{35}$ (Supplementary Fig. 3c). To assess other immune responses, we measured macrophage polarization, cytokine secretion profiles and phagocytic activity (Fig. $3 \mathrm{c}-\mathrm{e}$ ). Polarization was measured in nonactivated (M0) macrophages infected with SARS-CoV-2 and markers compared with those that were either differentiated into a pro-inflammatory (M1) state using M-CSF, lipopolysaccharide (LPS) and IFN- $\gamma$ or a wound-healing M2 state using macrophage colony-stimulating factor (M-CSF) and interleukin-4 (IL-4) (Fig. 3c). SARS-CoV-2-infected macrophages displayed a strong M1-like pro-inflammatory phenotype (Fig. 3c). We also measured induction of a selected set of cytokines that have been reported to be altered in SARS-CoV-2 infection. Secretion of pro-inflammatory cytokines such as IL-1 $\beta$, monocyte chemoattractant protein 1 (MCP1) and IL-6 was significantly upregulated in SARS-CoV-2 infection. On the other hand, that of types I and II IFNs was significantly downregulated, recapitulating the early events in the immunopathology of patients with COVID-19 (Fig. 3d). Similarly, the phagocytic activity of SARS-CoV-2-infected macrophages resembled the reduced activity observed in M1 but not M2 macrophages (Fig. 3e). To determine whether these cytokines followed the same secretory mechanism as ISG15, we depleted secretory autophagosomal components (LC3, ULK1 and STX4) by Dicer-substrate small interfering RNA (DsiRNA) treatments. Depletion of STX17 was performed to inhibit degradative autophagy. Control and depleted cells were challenged with SARS-CoV-2, and released cytokines were measured as described above. Although all cytokines displayed significant decreases in released amounts, none of them were completely abolished, suggesting that they are probably secreted via multiple pathways (Fig. 3f). Interestingly, secretion of TNF- $\alpha$ was not affected in the autophagy-depleted cells, suggesting 
a
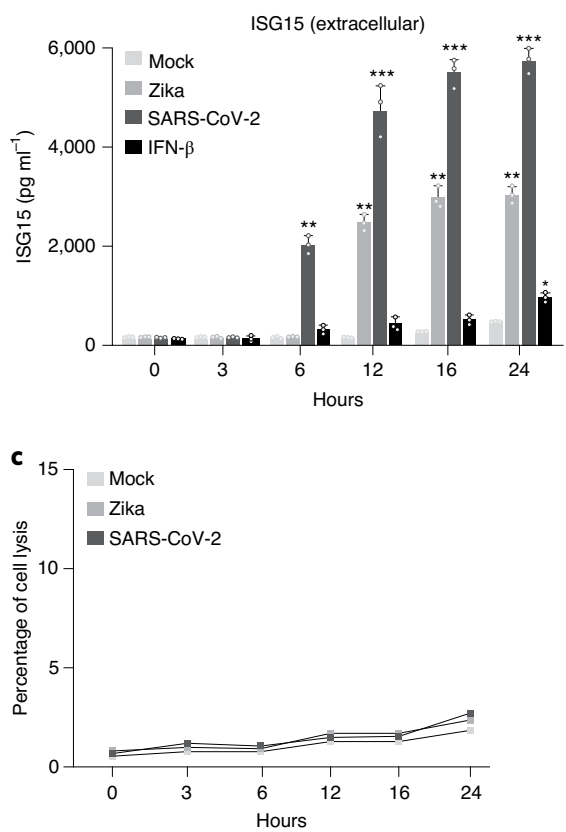

b

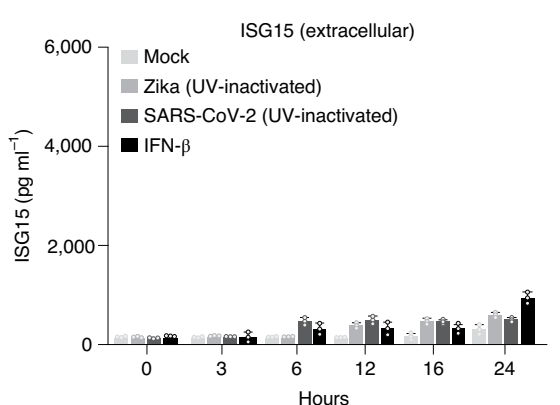

d

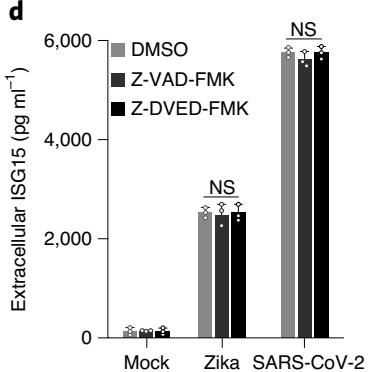

e

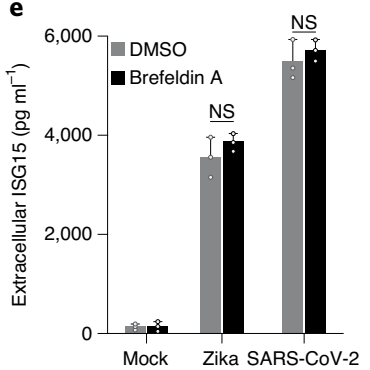

f

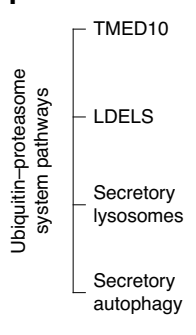

g

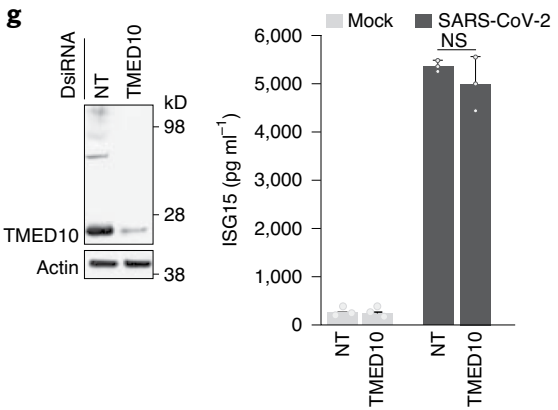

h

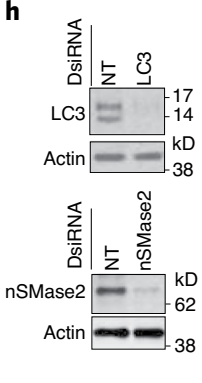

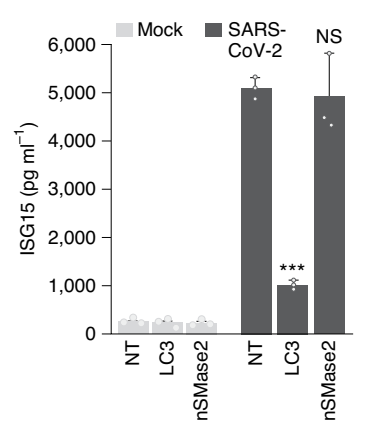

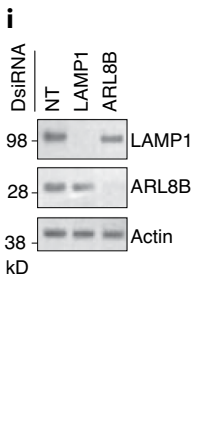

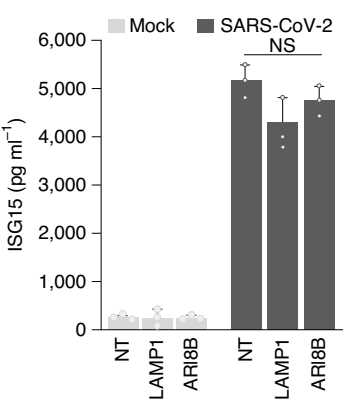

j

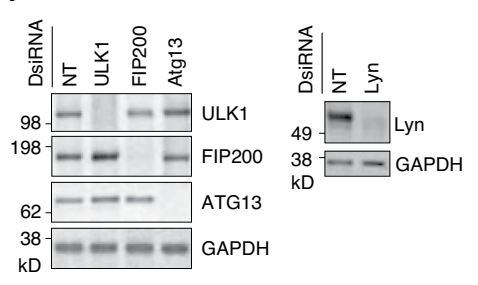

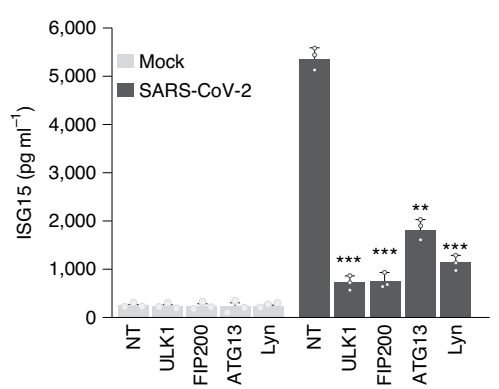

k

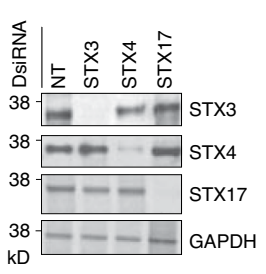

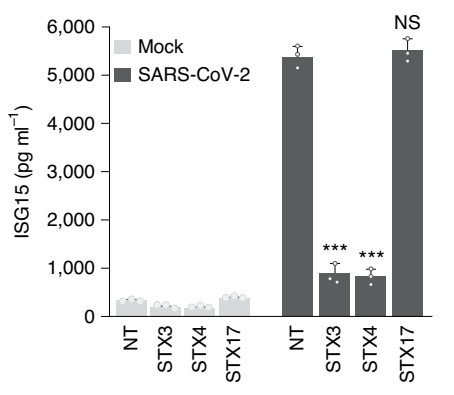

Fig. 2 | ISG15 is secreted from virus-infected macrophages via an unconventional, LC3-derived secretory pathway. a,b, Macrophages were infected with either Zika or SARS-CoV-2 at an MOI of 2 (a) or with an equivalent dose of UV-inactivated Zika or SARS-CoV-2 (b) virus. Changes in extracellular levels of ISG15 were quantified by ELISA. c, LDH assay was performed using the LDH-Cytotoxicity Colorimetric Assay Kit II (BioVision) to estimate cell death based on the amount of LDH leakage into the cell culture media at the indicated time points post-infection according to the manufacturer's protocol. $\mathbf{d}$, The caspase inhibitors Z-YVAD-FMK and Z-DEVD-FMK were added to virus-infected macrophages ( $1 \mathrm{~h}$ post-infection) and ISG15 secretion was measured $24 \mathrm{~h}$ post-infection. e, Brefeldin A ( $5 \mu \mathrm{M})$ or dimethyl sulfoxide (DMSO) alone was added $1 \mathrm{~h}$ post-infection and ISG15 secretion was measured $24 \mathrm{~h}$ post-infection. f, Potential pathways of unconventional protein secretion. g-k, Depletion of TMED10 (g), LDELS components (LC3; nSMase2) (h) and secretory lysosomal proteins (LAMP1, ARL8B) (i), early autophagy components (ULK1 complex, Lyn kinase) (j) and SNARE proteins of the secretory autophagy pathway ( $\mathbf{k}$ ) were performed by DsiRNA treatment and verified by immunoblotting. NT and depleted cells were infected with SARS-CoV-2 $(\mathrm{MOI}=2 ; 24 \mathrm{~h})$ and secretion of ISG15 measured by ELISA. a-k. The error bars represent the means \pm s.d. of three biologically independent experiments. ${ }^{\star} P<0.05,{ }^{\star \star} P<0.01,{ }^{\star \star \star} P<0.001$ by two-sided Mann-Whitney U-test versus mock-infected cells.

that all cytokines are not secreted via the same route. Collectively, these data indicate that infection by SARS-CoV-2 results in aberrant macrophage responses, downregulating antigen presentation and triggering secretion of inflammatory cytokines, which might underpin the consistent symptoms of lymphopenia and cytokine storm observed in patients with COVID-19. 
a

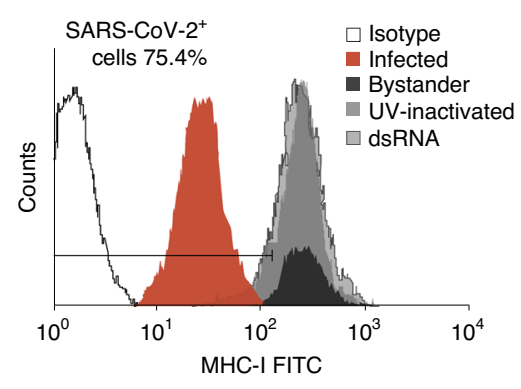

b

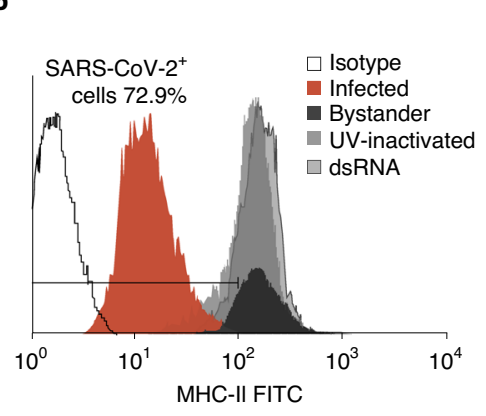

C

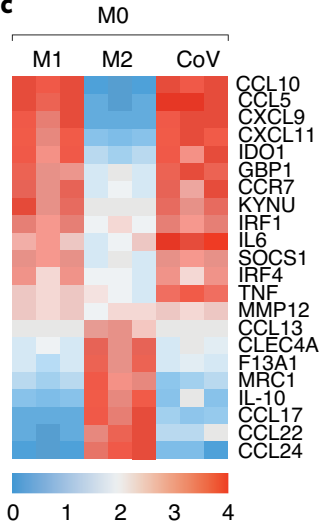

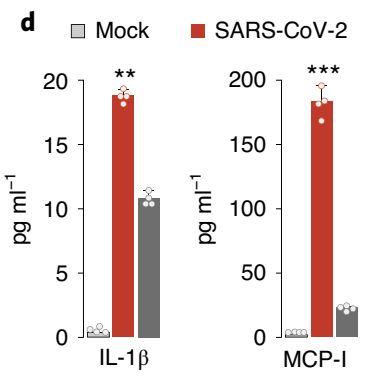
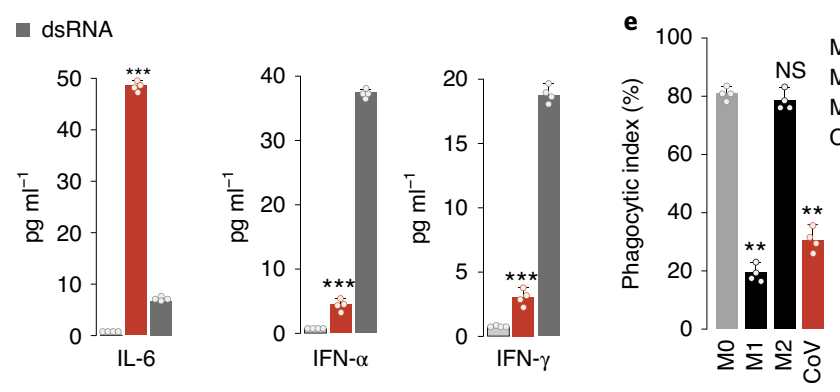

M0: M-CSF

f
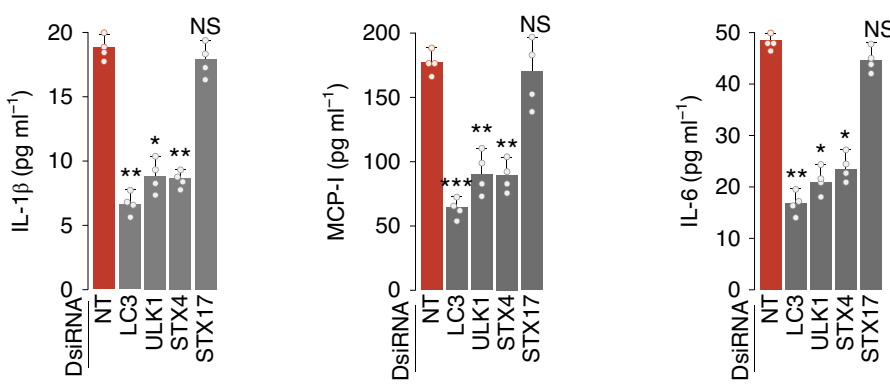

Fig. 3 | Immune dysfunction in SARS-CoV-2-infected macrophages. a, Surface staining of MHC-I in macrophages infected with SARS-CoV-2 (MOI=2, $24 \mathrm{~h}$ ). Controls included were antibody isotype, UV-inactivated virus and dsRNA. Cells were gated on viral $\mathrm{N}^{+}$(in red; $75 \%$ of population) and surface MHC-I. Uninfected cells (bystander; viral $\mathrm{N}^{-}$) are depicted in black. FITC, fluorescein isothiocyanate. b, Surface staining of MHC-II in macrophages infected with SARS-CoV-2 ( $\mathrm{MOI}=2,24 \mathrm{~h}$ ). Cells were gated on viral $\mathrm{N}^{+}$(in red; $73 \%$ of population) and surface $\mathrm{MHC}^{-I^{+}}$cells. The uninfected population (bystander; viral $\mathrm{N}^{-}$) is depicted in black. Controls included were antibody isotype, UV-inactivated virus and dsRNA-treated cells. c, MO macrophages were stimulated to $\mathrm{M} 1$ or $\mathrm{M} 2$ by differentiating for $48 \mathrm{~h}$ in the presence of M-CSF + LPS + IFN- $\gamma$ and M-CSF + IL-4, respectively, or infected with SARS-CoV-2 (MOI=2, $48 \mathrm{~h})$. Expression of key markers of polarization was measured by RT-qPCR. $\mathbf{d}$, Secretion of the indicated cytokines was measured using cytometric bead array according to the manufacturer's guidelines and flow cytometry. The error bars represent the mean \pm s.d. ${ }^{\star \star} P<0.01$, ${ }^{\star \star \star} P<0.001 ; n=4$ biologically independent experiments. e, Quantification of phagocytosis of M1- or M2-stimulated phagocytes was compared with SARS-CoV-2-infected macrophages $(\mathrm{MOI}=2,48 \mathrm{~h})$. The error bars represent the mean \pm s.d. ${ }^{\star} P<0.05,{ }^{\star \star} P<0.01,{ }^{\star \star \star} P<0.001,{ }^{\star \star \star \star} P<0.0001 ;$ two-way ANOVA with Tukey's multiple comparison test, $n=4$ biologically independent experiments. $\mathbf{f}$, Cells depleted of secretory autophagosomal components (LC3, ULK1, STX4) were challenged with SARS-CoV-2 and the indicated cytokines measured by cytometric bead array; the error bars represent the mean \pm s.d. ${ }^{\star} P<0.05,{ }^{\star \star} P<0.01,{ }^{\star \star \star} P<0.001,{ }^{\star \star \star \star} P<0.0001 ; n=4$ biologically independent experiments by two-tailed Man-Whitney $U$-test compared with control cells.

Substrate deISGylation drives pro-inflammatory cytokine responses. To decouple the role of ISGylation from free ISG15 in virus-infected macrophages, we systematically knocked down ISG15, UBE1L, HERC5 and USP18 by transfecting macrophages with DsiRNA $72 \mathrm{~h}$ before infecting with either Zika or SARS-CoV-2 virus as specified. The knockdown efficiency of ISG15 and its modifying enzymes was verified in type I IFN-treated cells by immunoblotting. The results confirmed that all DsiRNA targets were significantly depleted compared to control cells (Fig. 4a and Supplementary Fig. 5a-c). In ISG15-depleted cells, as predicted, both free and ISGylated forms were downregulated, whereas in UBE1L and HERC5 knockdown cells, only the conjugated forms were downregulated (Fig. $4 \mathrm{~b}$ and Supplementary Fig. $5 \mathrm{~d}$ ). USP18 is the cellular deISGylating enzyme and a negative regulator of type I IFN response; as anticipated, we observed an upregulation of ISGylated material in USP18-depleted macrophages (Fig. 4b). Interestingly, depleting HERC5 or UBE1L did not result in increase in mono ISG15 in the lysates of infected cells compared to IFN-treated cells, which is probably due to increased secretion of ISG15. Depletion of either ISG15, ISGylating enzymes or USP18 did not have any significant effect on the replication of Zika (Fig. 4c), SARS-CoV-2 (Fig. 4e) or influenza (Supplementary Fig. 5e) in macrophages. On the other hand, depletion of ISGylation alone, but not ISG15, stimulated secretion of pro-inflammatory cytokines, particularly 

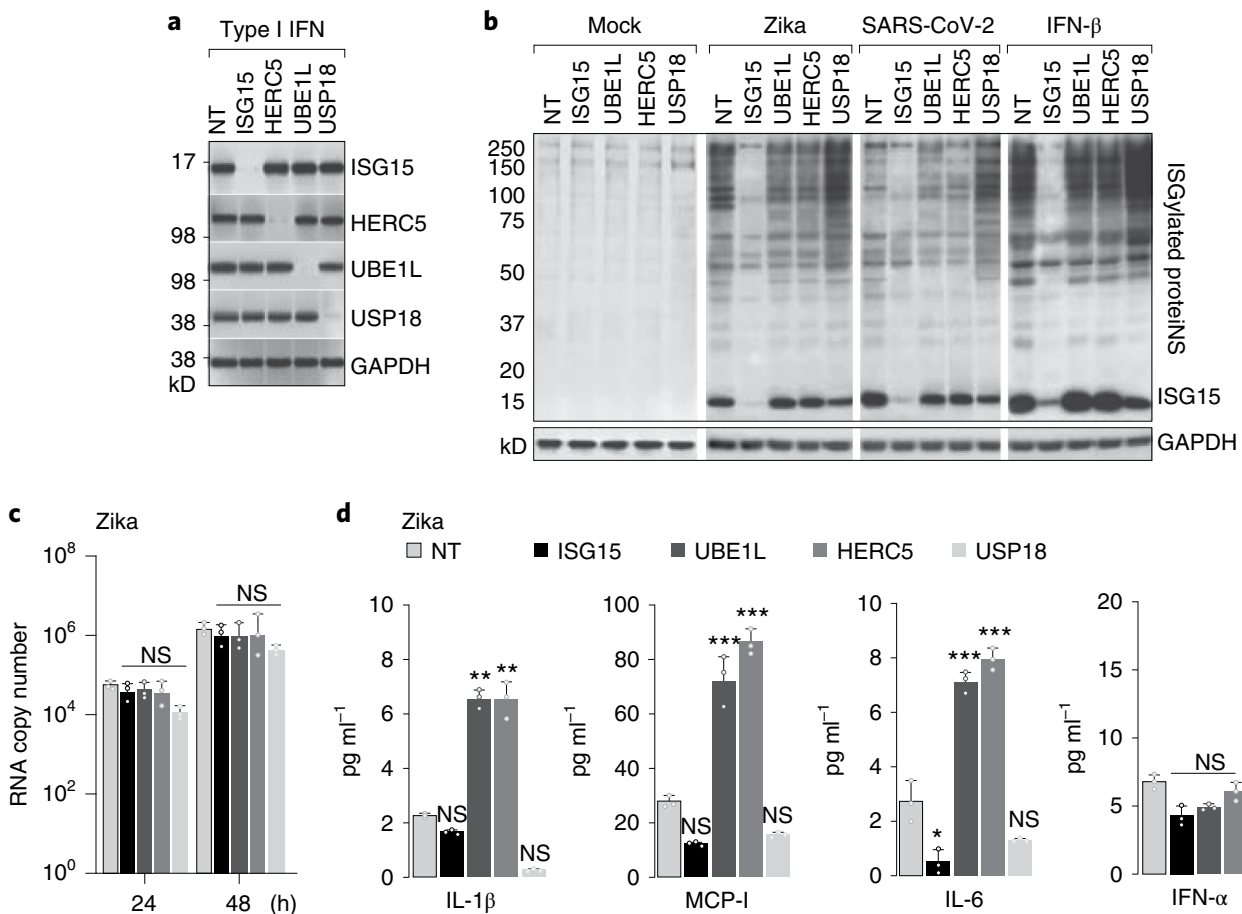

\section{d Zika} $\square$ NT
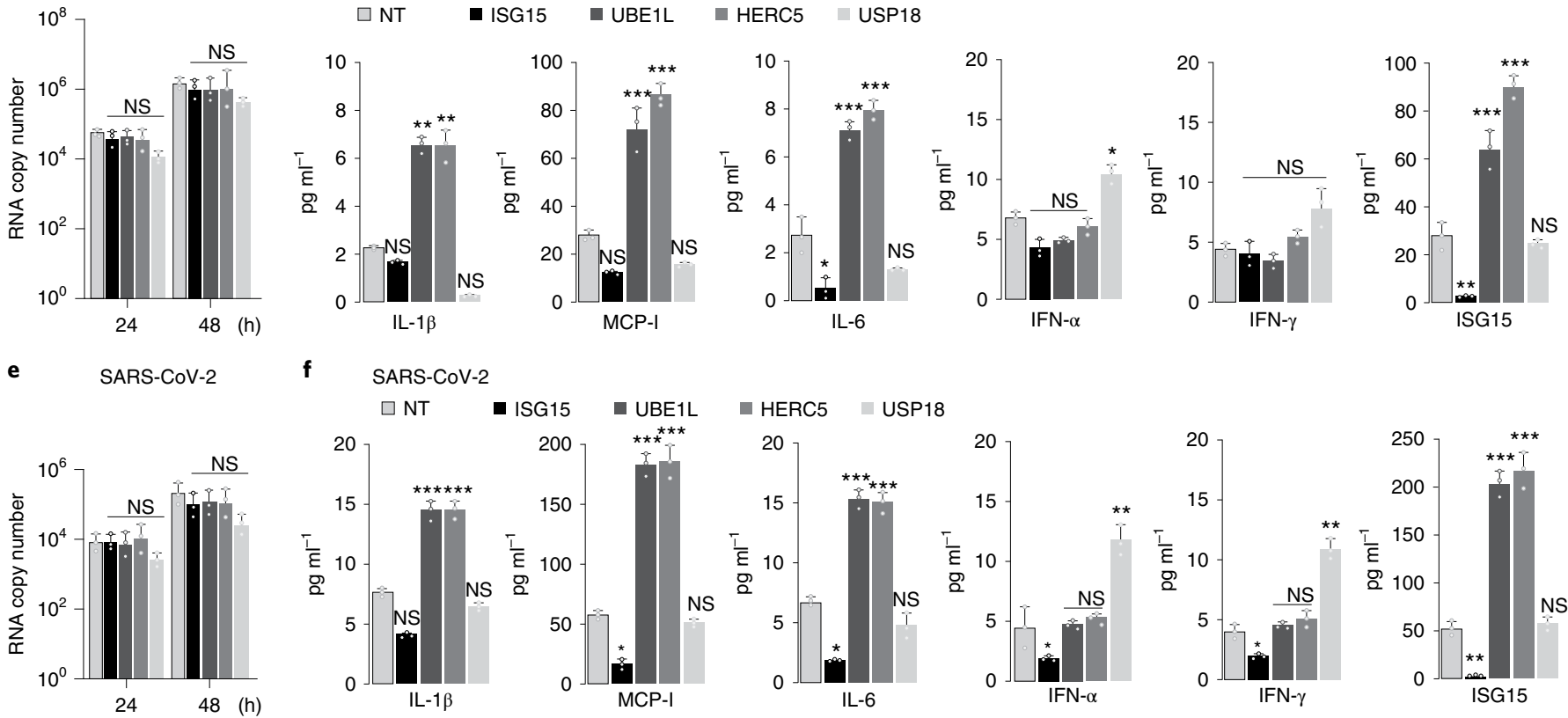

f SARS-CoV-2
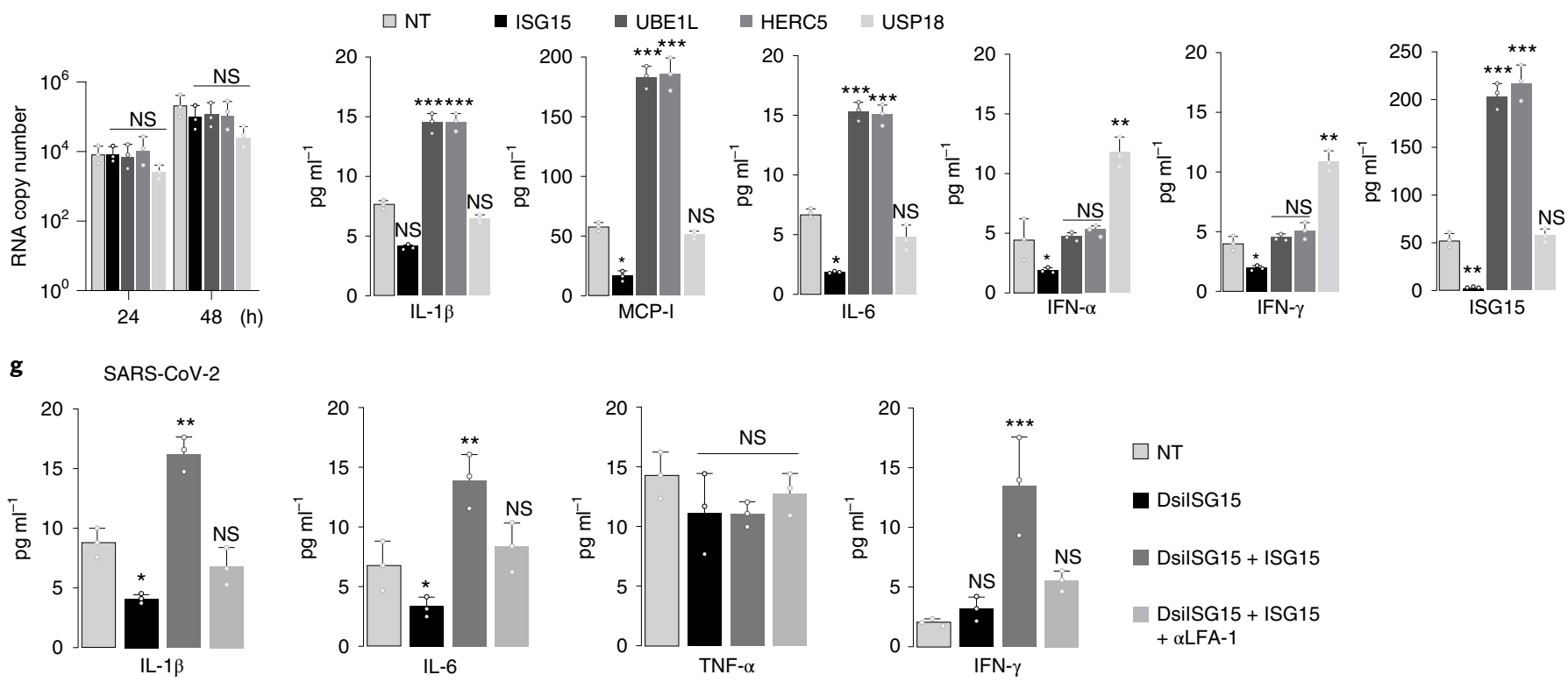

$\square$ NT

DsilSG15

DsilSG15 + ISG15

DsilSG15 + ISG15

$+\alpha$ LFA -1

Fig. 4 | Role of free versus conjugated ISG15 on viral replication and cytokine secretion. a, Macrophages were transfected with ISG15, UBE1L, HERC5 or USP18 DsiRNA for $72 \mathrm{~h}$; depletion was verified in type I IFN-treated cells by immunoblotting. b. Cellular ISGylation was measured in Zika- and SARS-CoV-2infected cells $(\mathrm{MOI}=2)$, $24 \mathrm{~h}$ post-infection. $\mathbf{a}, \mathbf{b}$, Immunoblots are representative of three biologically independent experiments. c, Total RNA was collected at the indicated time intervals from Zika-infected cells; quantifications of absolute copy number were done by RT-qPCR using universal viral RNA-specific primers. Data are displayed as the mean \pm s.e.m. of three biologically independent experiments. ${ }^{\star} P<0.05$ by Mann-Whitney $U$-test versus control. $\mathbf{d}$, The indicated cytokines were quantified by cytometric beads assay from macrophages transfected with either NT, ISG15, UBE1L, HERC5 or USP18 DsiRNA for $72 \mathrm{~h}$ before infection. Free ISG15 was determined by ELISA. Data are displayed as the mean \pm s.e.m. of three biologically independent experiments. ${ }^{\star} P<0.05$, ${ }^{\star \star} P<0.01,{ }^{* \star} P<0.001$ by two-tailed Mann-Whitney $U$-test versus control. e, Total RNA was collected at the indicated time intervals from SARS-CoV2-infected cells; quantifications of absolute copy number were done by RT-qPCR using viral RNA-specific primers. Data are displayed as the mean \pm s.e.m. of three biologically independent experiments. ${ }^{\star} P<0.05$ by Mann-Whitney $U$-test versus control. $\mathbf{f}$, The indicated cytokines were quantified by cytometric beads assay from macrophages transfected with either NT, ISG15, UBE1L, HERC5 or USP18 DsiRNA for $72 \mathrm{~h}$ before infection. Free ISG15 was determined by ELISA. Data are displayed as the mean \pm s.e.m. of three biologically independent experiments. ${ }^{\star} P<0.05$ by two-tailed Mann-Whitney $U$-test versus control (NT cells). g, Macrophages were treated with ISG15 DsiRNA and infected with SARS-CoV-2 $(\mathrm{MOI}=2)$ in media supplemented with exogenous purified ISG15 $\left(1 \mu \mathrm{g} \mathrm{ml}^{-1}\right)$; cells were either untreated or pretreated with anti-LFA-1 inhibitory antibody $(500 \mathrm{ng})$ for $1 \mathrm{~h}$ before infection. The indicated cytokines were quantified by cytometric beads assay. The error bars represent the mean \pm s.d. ${ }^{\star} P<0.05,{ }^{\star \star} P<0.01$ by two-tailed Mann-Whitney $U$-test versus control cells ( $n=3$ biologically independent experiments). 
MCP-1, IL-6 and IL-1 $\beta$ and free ISG15 from virus-infected cells in general (Fig. 4d,f and Supplementary Fig. 5f) but most prominently from SARS-CoV-2-infected cells (Fig. 4f). Interestingly, production of types I and II IFNs from Zika- and SARS-CoV-2-infected macrophages displayed the reverse effect. Cells depleted in ISG15 conjugating enzymes did not have any significant effect on type I or II IFN production, whereas USP18-depleted cells displayed a modest increase (Fig. 4f). To further decouple the effect of conjugated versus free ISG15, we infected ISG15-depleted cells with SARS-CoV-2 and treated them with purified exogenous ISG15 to measure its effect on cytokine secretion (Fig. 4g). While ISG15-depleted cells displayed reduced secretion of cytokines, treatment with exogenous ISG15 triggered increased production of IL- $1 \beta$ and IL- 6 ; however, secretion of TNF- $\alpha$ was unaffected, suggesting that different cytokines might be secreted via distinct routes and free ISG15 possibly triggers a subset of them. Interestingly, secretion of IFN- $\gamma$ was also induced on exogenous ISG15 treatment, unlike WT cells. The phenomenon of increased cytokine secretion by exogenous ISG15 treatment was partially inhibited by pretreating cells with anti-lymphocyte function-associated antigen 1 (LFA-1), which was previously reported as the receptor for secreted ISG15 (ref. ${ }^{26}$ ), supporting the role of free ISG15 in triggering inflammatory responses in a paracrine manner (Fig. 4g). Depletion of ISG15 and UBE1L/HERC5 but not USP18 also resulted in reduced phagocytic activity in influenza-infected macrophages, indicating that ISGylation is important for this effector function of macrophages as reported previously (Supplementary Fig. 6a,b). Collectively, these data indicate that skewing the ratio toward a higher proportion of free ISG15 to its conjugated form drives hyperproduction of at least a subset of pro-inflammatory cytokines often detected in severe respiratory infections.

SARS-CoV-2 PLpro recapitulates aberrant macrophage phenotypes. Several viruses including coronaviruses encode deubiquitylases and deISGylases in their genome. To evaluate whether expressing the viral deISGylase itself was sufficient to induce aberrant macrophage responses, we expressed WT and catalytically inactive SARS-CoV-2 PLpro in macrophages (Fig. 5a,b). WT and mutant USP18 was expressed in parallel as controls. We measured cellular ISGylation on type I IFN treatment in cells expressing

Fig. 5 | Dysregulation of antigen presentation and interferon response in macrophages expressing SARS-CoV-2 PLpro. a, b, Schematic of SARS-CoV-2 PLpro (WT (a) and mutant (b)) and their expression in macrophages verified by immunoblotting. c, Bulk ISGylation in type I IFN-treated macrophages expressing either the empty vector, WT PLpro or mutant PLpro in a dose-dependent manner. b,c, Immunoblots are representative of three biologically independent experiments. $\mathbf{d}, \mathbf{e}$, Surface staining of MHC-I in macrophages expressing either WT hemagglutinin (HA)-tagged PLpro (d) or the catalytic mutant HA-tagged PLpro (C111A) (e) of SARS-CoV-2. Cells were then treated with dsRNA to induce surface expression of MHC-I. For both samples (WT, mutant C111A) >90\% of cells stained positive for HA-tagged PLpro and dsRNA. $\mathbf{f}, \mathbf{g}$, Same as $\mathbf{d}, \mathbf{e}$ in cells expressing USP18 (WT (f) or C64R/C65R mutant $(\mathbf{g})$ ). $\mathbf{h}$, Secretion of the indicated cytokines was measured using cytometric beads assay according to the manufacturer's guidelines and flow cytometry. The error bars represent the mean \pm s.d. from four independent experiments. Data are displayed as the mean \pm s.d. ${ }^{\star} P<0.05$ by two-tailed Mann-Whitney $U$-test versus control (empty vector matched cells). i, Supernatants from the cells described in $\mathbf{h}$ were collected and ISG15 was quantified by ISG15 sandwich ELISA. All data are displayed as the mean \pm s.d. of four independent experiments. ${ }^{\star} P<0.05$ by two-tailed Mann-Whitney $U$-test versus control cells. j, ISG15 levels in the plasma samples collected from the patients with COVID-19 at their first week of disease onset. The error bars represent the mean \pm s.d. ${ }^{*} P<0.05$ by two-tailed Mann-Whitney $U$-test versus healthy donors ( $n=38$ for patients and $n=14$ for healthy donors). either the empty control vector or those expressing either the WT or mutant variants of PLpro. Dose-dependent expression of the WT but not the mutant PLpro resulted in hydrolysis of bulk cellular ISGylation and a concomitant increase in free ISG15 in type I IFN-treated cells, indicating that it is indeed an active deISGylase (Fig. 5c). Expression of PLpro alone did not induce expression of cellular USP18, suggesting that cellular deISGylation, at least in PLpro-expressing cells, was specifically due to PLpro activity (Fig. 5b). However, as the mRNA levels of USP18 increase in infected cells, it is likely that partial deISGylation occurs via USP18 function on infection. A recent study reported that USP18 decreases antigen presentation in cancer cells ${ }^{36}$; a similar phenomenon may well contribute to the immune dysregulation in virus-infected cells. a

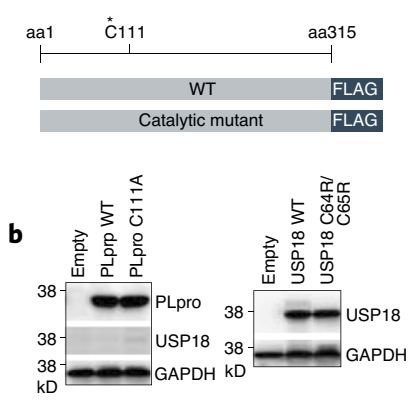

d PLpro WT $^{+}$

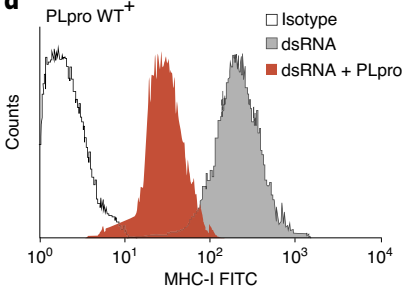

f USP 18 WT+

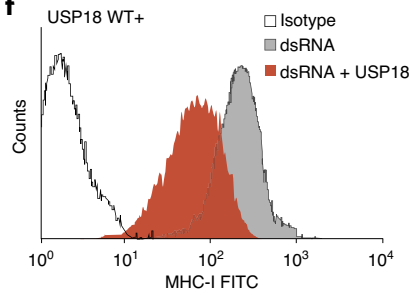

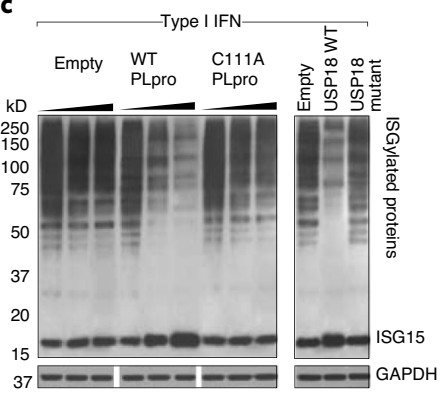

e
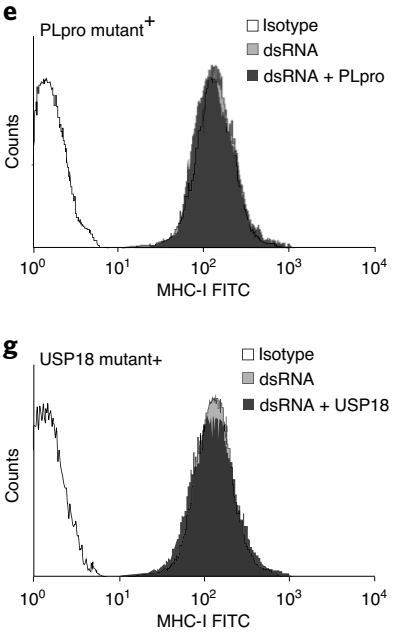

h

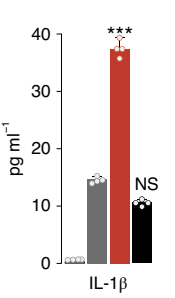

$\square$ Control $\square$ dsRNA $\square$ dsRNA + PLpro WT $\square$ dsRNA + PLpro C111A
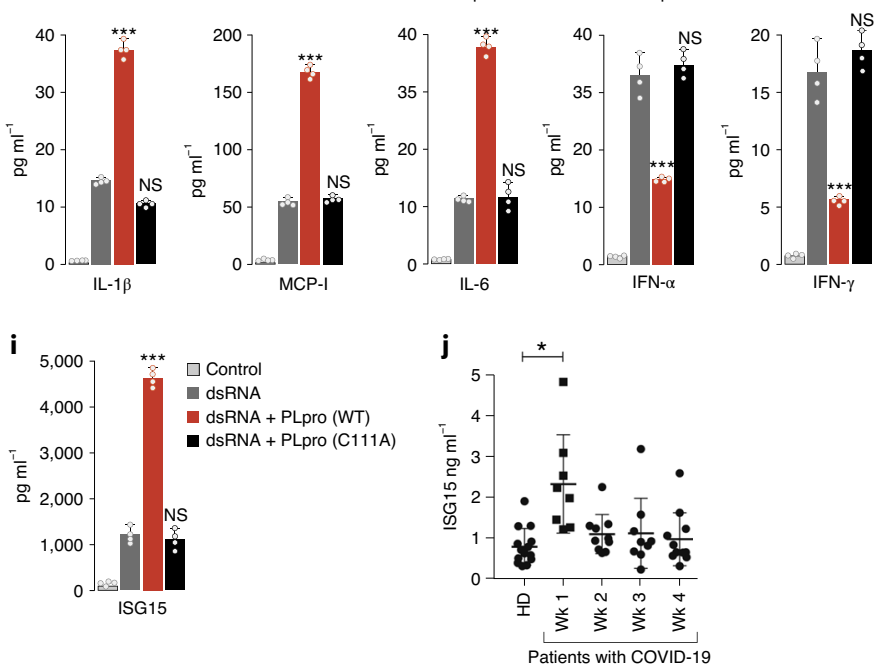
To investigate whether the PLpro enzyme was sufficient to alter macrophage responses, we measured the surface expression of MHC-I and secretion of the panel of cytokines described in Fig. 4 in cells transfected with dsRNA. Expression of the WT but not the mutant PLpro recapitulated downregulation of MHC-I presentation (Fig. 5d,e). Expression of the WT and catalytically mutant variants of USP18 - the cellular deISGylase - also confirmed the ISGylation-dependent downregulation of MHC-I (Fig. 5f,g). PLpro-expressing cells displayed increased secretion of pro-inflammatory cytokines such as MCP-1, IL- 6 , TNF- $\alpha$ and IL- $1 \beta$ along with free ISG15, and attenuated secretion of types I and II IFNs (Fig. 5h,i). To test these findings in clinical settings, we collected serum samples from patients, who also displayed increased amounts of free ISG15 at their first week of disease onset (Fig. 5j). Collectively, the results suggest that the deISGylating activity encoded by SARS-CoV-2 can disrupt early immune responses in macrophages, which likely contribute to the lymphopenia and cytokine storm that often accompany severe COVID- 19 .

SARS-CoV-2 PLpro deISGylates glycolytic enzymes. To further characterize the function of CoV-2 PLpro, we bacterially expressed and purified both the WT and C111A mutant variants of PLpro (Fig. 6a). The purified enzymes were tested on lysates from macrophages and HeLa cells treated with type I IFN. In both cases, the WT enzyme substantially hydrolyzed ISGylated proteins (Fig. 6b). We determined their substrate preference using propargyl amide (PA) activity-based probes for both ubiquitin and ISG15. As previously reported, PLpro displayed higher activity toward the ISG15 probe compared to ubiquitin (Fig. 6c).

To delineate the effects of free and conjugated ISG15, we performed a proteome-wide analysis of host ISG15 sites targeted by SARS-CoV-2 PLpro. Our search strategy was based on the original ISG15-GlyGly peptidomics tool ${ }^{37}$. We used trypsin digestion to reveal diglycines on the modified lysine residues of ISGylated proteins to enrich modified peptides and locate ISG15 modification sites on target proteins by liquid chromatography-tandem mass spectrometry (LC-MS/MS). However, because ubiquitin and NEDD8 leave the same diglycine adduct after trypsin digestion, we used $I S G 15^{-/}$cells as a control to distinguish bona fide ISGylation sites.

We applied the ISG15-GlyGly peptidomics tool on HeLa cells producing high levels of ISGylated proteins on stimulation with type I IFN. Lysates from WT and ISG15 1 cells were incubated with recombinant WT or mutant PLpro. Treatment with WT PLpro markedly reduced the levels of ISG15 conjugates compared to untreated or mutant PLpro-treated samples (Fig. 6d). In addition, we observed a minor decrease in ubiquitination levels after WT PLpro treatment, which is in line with our activity-based assay (Fig. 6c) and with previous reports showing weak activity of PLpro toward ubiquitin ${ }^{38}$. PLpro-treated samples were trypsin-digested to generate diglycine-modified peptides that were enriched by immunoprecipitation and quantified by LC-MS/MS. After statistical analysis and unsupervised hierarchical clustering, significantly regulated sites were grouped into three major clusters (Fig. 6e and Supplementary Table 1). Replicate cell cultures clustered together by genotype and treatment, indicating the high reproducibility of our approach. In total, we uncovered 276 ISGylation sites on 181 human proteins that were induced on stimulation with type I IFN (Fig. 6e, clusters 1 and 2). These sites were mostly absent in ISG15 1 control cells, thereby marking them as bona fide ISGylation sites (Fig. 6e, indicated in gray). Interestingly, more than half of the identified ISGylation sites were targeted by PLpro as indicated by their absence in the WT PLpro-treated samples (Fig. 6e, cluster 1a). In addition, a small subset of ISG15 sites was only partially removed by the viral protease, which might be the result of low enzyme:substrate ratios used during PLpro treatment (Fig. 6e, cluster 1b). Of note, baseline presence of some of these sites in the ISG15\% cells might also result from coregulation by ubiquitin. Finally, we found 20 ubiquitination sites on 16 proteins that were uniquely upregulated in $I S G 15^{-/}$cells on type I IFN treatment, with none of these sites targeted by PLpro (Fig. 6e, cluster 3). To verify that proteome alterations in the ISG15 $5^{-/}$cells did not lead to false positive identifications of ISG15 sites (for example, by downregulation of ubiquitinated proteins in these cells), we checked shotgun data of the input to highlight differentially regulated proteins between WT and ISG15 ${ }^{-/}$cells on type I IFN treatment (Supplementary Fig. 7 and Supplementary Table 2). Comparison of protein intensities between WT and $I S G 15^{-/}$samples revealed 282 significantly regulated proteins, irrespective of the treatment with PLpro (Supplementary Fig. 7 and Supplementary Table 3). Many of these proteins were upregulated in $I S G 15^{-/}$cells, in line with previous reports showing an amplified type I IFN response in cells from ISG $15^{-1-}$ patients ${ }^{39}$. Interestingly, many of the upregulated ubiquitination sites in $I S G 15^{-/}$cells (cluster 3) were also found on these upregulated ISGs (Supplementary Fig. 7). By contrast, only 17 of the identified ISG15 sites (listed in Supplementary Table 1) were present on significantly regulated proteins, which precludes any major effect of genotype on ISG15 site identification (Supplementary Fig. 7). Collectively, our approach led to the high-confidence identification of 118 ISGylation sites on 95 proteins as targets of the SARS-CoV-2 protease PLpro. To validate some of the top-scoring candidates, we radiolabeled macrophages expressing either the WT or mutant variants of PLpro with $\left[{ }^{35} \mathrm{~S}\right]$ cysteine/methionine. Endogenous proteins were immunoprecipitated using specific antibodies, resolved by gel electrophoresis and their ISGylated forms detected by autoradiography (Fig. 6f). In line with the MS data, we confirmed the ISGylation and PLpro-mediated deISGylation of glycolytic enzymes and TAP-transporter associated with antigen processing. Interestingly, only a very minor fraction of IRF3, which was previously reported to be the primary target of PLpro was ISGylated.

Modification of glycolytic enzymes by ISG15 suppresses proinflammatory gene expression in adipocytes ${ }^{40}$. This phenomenon is linked to the production of macrophage-derived cytokines, such as TNF and IL-6, and pattern recognition receptors. ISGylation of glycolytic enzymes suppresses the glycolytic flux, resulting in attenuated expression of inflammatory genes. Expression of the cellular deISGylase USP18 rescued these defects. Our ISGylome data indicate that this is also the case with SARS-CoV-2 PLpro. Many of the highest-scoring candidates for deISGylation were enzymes of the glycolytic pathway, indicating that PLpro very likely reverses the ISG15-dependent suppression of inflammation in infected cells and drives the M1 pro-inflammatory phenotype during SARS-CoV-2 infection.

SARS-CoV-2-infected macrophages secrete pro-inflammatory cytokines. The aberrant cytokine responses from PLpro-expressing cells prompted us to systematically analyze the ISG15-dependent secretome from SARS-CoV-2-infected macrophages and compare that with IFN- $\gamma$-treated macrophages. We performed a quantitative MS-based proteomic analysis of the extracellular protein profile (secretome) of SARS-CoV-2-infected and IFN- $\gamma$-treated macrophages using established strategies of label-free quantitation. We selected $24 \mathrm{~h}$ post-SARS-CoV- 2 infection or IFN- $\gamma$ treatment as the time point for analyses in macrophages expressing nontargeting (NT) DsiRNA or those targeting ISG15 or UBE1L. We defined the secretome as proteins released via all mechanisms, including the classical, nonclassical and exosomal pathways. Using LC-MS/MS and the MaxQuant proteomics software package for computational analyses, we detected relative protein abundances in the conditioned media of control, SARS-CoV-2-infected or IFN- $\gamma$-treated macrophages. For increased confidence in the protein identification numbers, we required that a protein be identified on the basis of at least two unique peptides and quantified in a minimum of two replicates. 
a

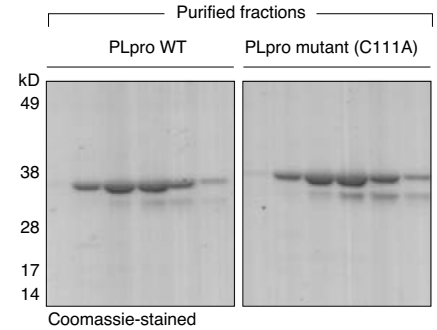

b

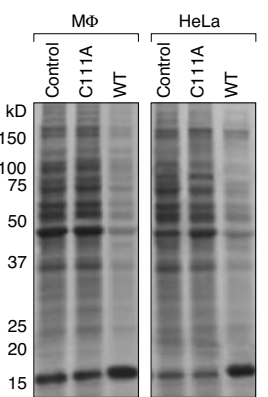

d

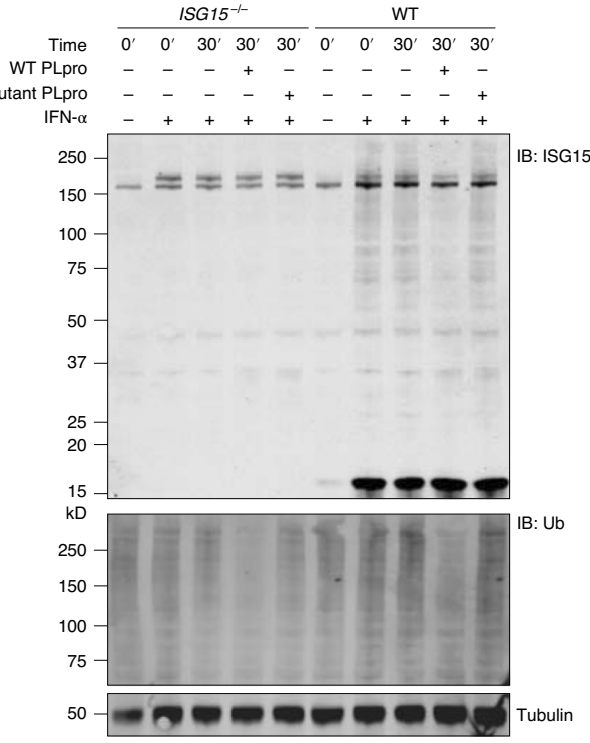

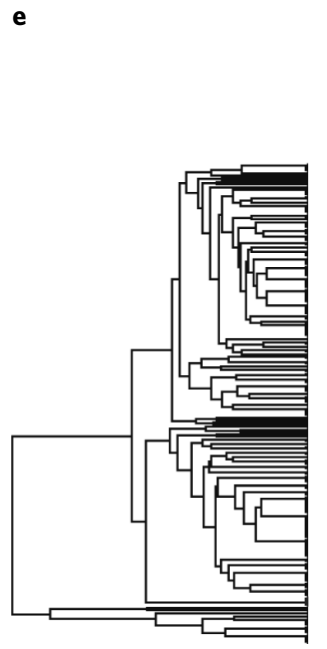
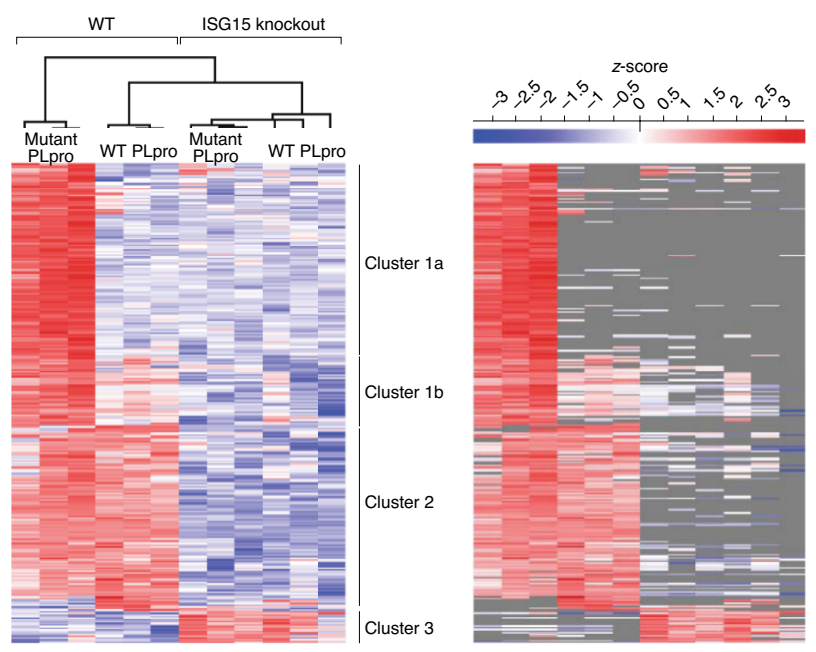

f
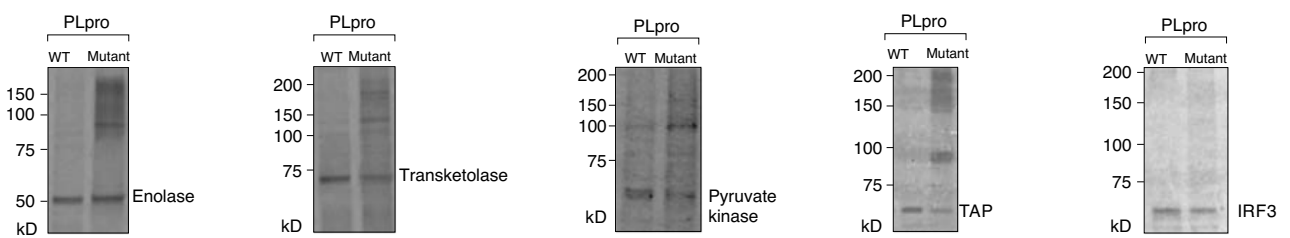

Fig. 6 | Identification of substrates delSGylated by SARS-CoV-2 PLpro. a, Coomassie-stained gel of fractions collected from size-exclusion chromatography for bacterially expressed and purified WT and mutant (C111A) SARS-CoV-2 histidine-tagged PLpro. b, Cell lysates from type I IFN-treated macrophages or HeLa cells were treated with buffer alone (control lanes), purified WT or mutant (C111A) PLpro. Lysates were resolved by gel electrophoresis and visualized by western blotting. c, Activity-based assay to determine deubiquitylating versus delSGylating activities of WT and C111A PLpro; $10 \mu \mathrm{M}$ of either HA-tagged Ub-PA or ISG15-PA were treated with the indicated concentrations of WT and mutant (C111A) PLpro for 30 min at room temperature. USP18 was used as a positive control for delSGylase activity. Reaction products were resolved by gel electrophoresis and visualized by Coomassie staining. d, Sample preparation for mass spectrometry to identify PLpro substrates. Western blot analysis of cellular lysates obtained from HeLa WT or ISG15 ${ }^{-1-}$ cells after $72 \mathrm{~h}$ of type I IFN stimulation and treatment with recombinant WT or mutant PLpro for 30 min. The same lysates were used for the actual ISG15-GlyGly peptidomics experiment. ISG15 and ubiquitin conjugates were visualized by immunoblotting with anti-ISG15 (IB:ISG15) and anti-ubiquitin (IB:UBQ) antibody, respectively. Tubulin- $\alpha$ served as a loading control and was detected with anti-tubulin- $\alpha$ antibody (IB:Tub- $\alpha$ ). a-d, Images are representative of three biologically independent experiments. e, Heatmap showing significantly regulated GlyGly(K) sites after unsupervised hierarchical clustering. Different genotypes (WT or ISG15 knockout) and protease treatments (WT or mutant PLpro) are indicated. The colors indicate upregulated (red) or downregulated (blue) sites. Right, The same heatmap is shown with the originally missing values depicted in gray. Three major clusters can be observed that contain the ISG15 sites targeted (cluster 1a/1b) or untargeted (cluster 2) by PLpro, or ubiquitin sites unaffected by PLpro treatment (cluster 3). f, Validation of identified hits in macrophages expressing either WT or C111A mutant variants of PLpro, radiolabeled with $\left.{ }^{35} \mathrm{~S}\right]$ cysteine/methionine and endogenous proteins immunoprecipitated with antibodies against the indicated proteins. Autoradiographs are representative of three biologically independent experiments.

We identified 489, 428 and 502 proteins in IFN- $\gamma$-treated NT, ISG15-deficient and UBE1L-deficient macrophages, whereas we identified 508, 485 and 544 proteins in SARS-CoV-2-infected macrophages (Fig. 7a). Principal component analysis (PCA) of the secretome response in NT, ISG15-deficient and UBE1L-deficient cells showed a clear separation of ISG15-deficient cells from NT 

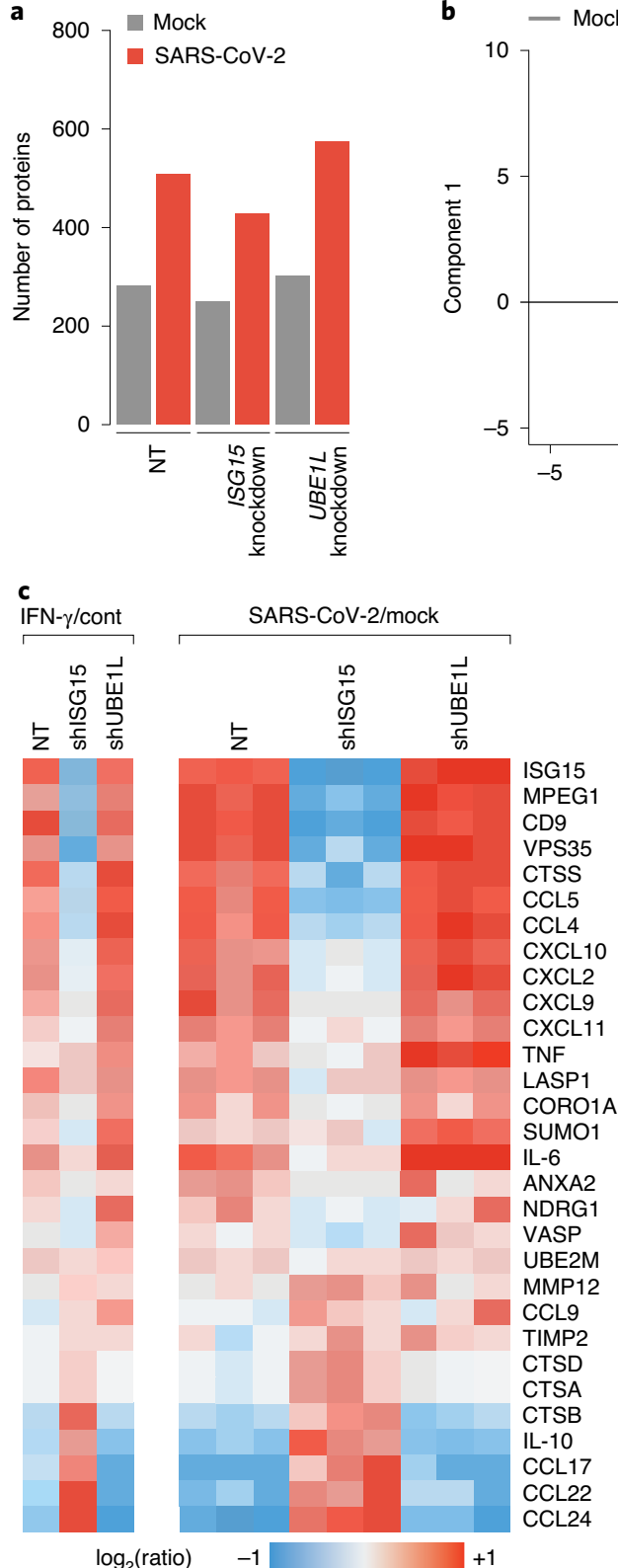
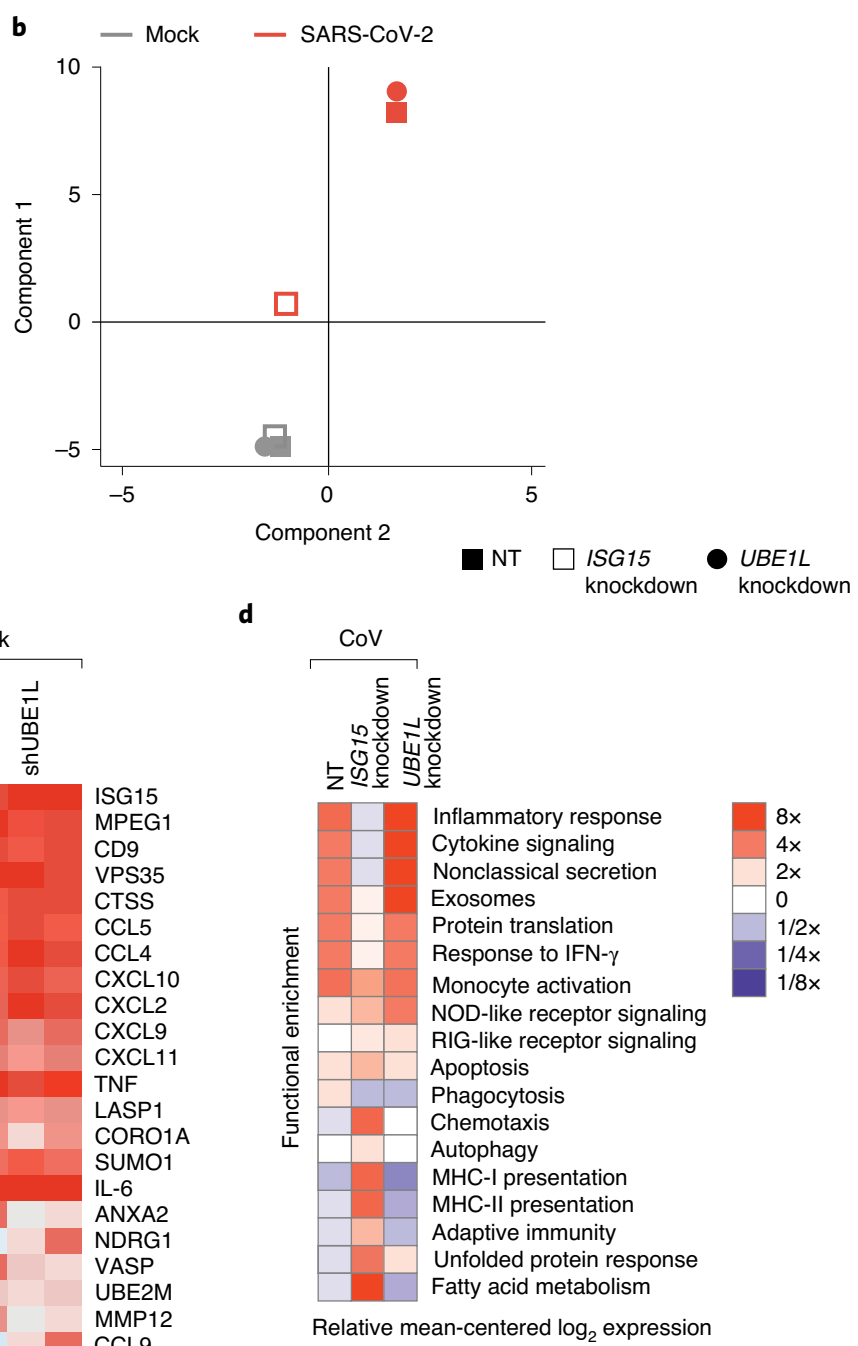

Fig. 7 | Quantitative analyses of ISG15-dependent responses in SARS-CoV-2-infected macrophages. a, iPSC-derived macrophages were either transfected with NT DsiRNA or those targeting ISG15 or UBE1L. Conditioned medium was collected from control, SARS-CoV-2-infected or IFN- $\gamma$-treated cells. Proteins were extracted from each of the samples, separated by SDS-PAGE and digested with trypsin for LC-MS/MS as described in the Methods. The total numbers of proteins were quantified in at least two biological replicates. b, PCA was performed using the Perseus software. The filled squares represent control cells (NT DsiRNA), the empty squares represent ISG15-depleted, the filled circles represent UBE1L-depleted. Uninfected cells are shown in gray and infected cells are shown in red. c, The heatmap represents the hierarchical clustering of the common proteins in the secretome for IFN- $\gamma$-treated or SARS-CoV-2-infected cells. The color key represents changes $\left(\log _{2}\right.$ scale) from dark blue indicating the largest decreases to red indicating the largest increases. d, Functional annotation of the common proteins identified in all samples was performed using the DAVID software.

and UBE1L-deficient cells, which clustered together (Fig. 7b). For the secretome datasets, the first 3 principal components captured 89\% (PC1: 51\%, PC2: 25\%, PC3: 13\%) variability in the data. Pairwise comparison showed the highest overlap between NT and UBE1L-deficient SARS-CoV-2-infected macrophages. We evaluated the responses of the common proteins identified in all the conditions. Hierarchical clustering analyses of these common proteins revealed that the secretome of NT and UBE1L-deficient cells clustered together while that of ISG15-deficient cells was significantly different from the others (Fig. 7c). Functional enrichment analyses revealed that the most prominent enrichment in the secretome of
SARS-CoV-2-infected cells were of the inflammatory responses, cytokine secretion, nonclassical secretory processes and exosomes, which strongly correlated specifically with UBE1L-deficient cells that inhibited the conjugated but not the extracellular free form of ISG15 (Fig. 7d). Collectively, our data indicate that altered free versus conjugated ISG15 results from the PLpro enzymatic function of SARS-CoV-2, which also triggers increased expression of pro-inflammatory genes and cytokines, skewing macrophages to the M1 state via perturbation of glycolytic flux. This phenomenon can be exacerbated by depleting the cellular ISGylation enzymes without affecting mono-ISG15. Free ISG15 released from infected 
macrophages can subsequently amplify cytokine secretion in a paracrine fashion via the LFA-1 receptor and Src family kinase activity, as shown previously. Therefore, these data provide a systematic overview of the core macrophage processes regulated by cellular ISG15 in response to SARS-CoV-2 infection.

\section{Discussion}

In vitro and in vivo studies have established that macrophages are one of the major determinants of pathogenesis during virus infections $s^{6,41-43}$, driven by the production of IFNs and IFN-stimulated genes. However, the role of ISG15 in its free or conjugated form in macrophage-mediated immune responses is not well studied. In this study, we compared the ISG15-dependent responses of human macrophages to influenza, Zika and SARS-CoV-2 virus infections. All these viruses transcriptionally upregulated ISG15 and ISGylating enzymes, which was accompanied by increased bulk protein ISGylation in influenza- and Zika- but not in SARS-CoV-2-infected cells. This is particularly intriguing as SARS-CoV-2 encodes for a PLpro, which is a putative deISGylase. SARS-CoV-2-infected cells displayed a skewed ratio of free versus conjugated ISG15, which was accompanied by heightened secretion of pro-inflammatory cytokines despite reduced IFN production and antigen presentation. Free ISG15 was secreted specifically from macrophages but not epithelial cells, which is consistent with its role in immune modulation. Secretion of ISG15 and pro-inflammatory cytokines was exacerbated by depleting the ISG15 conjugating enzymes UBE1L or HERC5, which prevented substrate ISGylation but not free ISG15. These data suggest that ISG15 regulates macrophage inflammatory responses either via protein ISGylation to inhibit secretory processes or via free ISG15 signaling that may induce cytokine production, or a combination of the two.

Our results demonstrate that the combined effect of deISGylation of metabolic enzymes and free form of ISG15 critically affect the global immune response of macrophages during virus infections, unlike respiratory tract epithelial cells where ISG15 primarily functions as an antiviral factor to limit viral replication ${ }^{17}$. Depletion of UBE1L or HERC5 that specifically prevented conjugated, but not free ISG15, stimulated secretion of pro-inflammatory cytokines from virus-infected macrophages, such as MCP-1, TNF and IL-6, all of which have been implicated in the cytokine storm caused by highly pathogenic influenza and severe COVID-19. MCP-1 (CCL2) and IL- 6 have been consistently found to be a predictor of severe pathogenesis in respiratory virus infections. Uncontrolled MCP-1 secretion has also been implicated in increasing the severity of inflammatory disorders of the lung and can regulate infiltration of immune cells, including monocytes, T cells and natural killer (NK) cells. Depletion of ISG15 followed by treatment with exogenous free ISG15 in SARS-CoV-2-infected cells recapitulated this phenotype for at least a subset of cytokines.

Several viruses encode deubiquitylating and deISGylating activities in their genome that can counter host antiviral immunity. SARS-CoV-2 itself encodes a PLpro that is a putative deISGylase $^{44}$. Our data indicate that infection by SARS-CoV-2 removes ISG15 modifications from cellular substrates, confirming its intrinsic deISGylating ability. Substrate deISGylation combined with increased free ISG15 was accompanied by hyperactivation of pro-inflammatory cytokines and reduced antigen presentation, both of which are key features of severe COVID-19. Expression of the WT but not catalytically inactive SARS-CoV-2 PLpro alone triggered dramatic cellular deISGylation, recapitulating these results. In vitro characterization of PLpro substrates further revealed that primarily metabolic enzymes of the glycolytic pathway are ISGylated during infection and targeted by the SARS-CoV-2 PLpro. ISGylation of glycolytic enzymes has been reported to suppress expression of thermogenic genes in obesity and polarization of macrophages to the M1 state. This feature can be reversed by loss of ISG15 or expres- sion of cellular deISGylase USP18. Besides, activation of the glycolytic pathway has been independently shown as critical for NK cell cytotoxicity and inflammatory response to cytomegalovirus infections ${ }^{45}$. Therefore, the widespread removal of ISG15 modifications from glycolytic enzymes indicates that the increased activation of this pathway drives the M1 pro-inflammatory phenotype in macrophages. Collectively, these data indicate that SARS-CoV-2 PLpro can perturb immune responses in macrophages, which may underpin the loss of $\mathrm{CD}^{+} \mathrm{T}$ lymphocytes and increased inflammation often seen in severe COVID-19 (ref. ${ }^{34}$ ).

The role of ISG15 in virus-infected macrophages underscore the importance that the conjugated and free forms play in driving immune responses. Extracellular free ISG15 can act as an adjuvant for $\mathrm{CD}^{+}$cytotoxic $\mathrm{T}$ cells and can also influence infiltration or activation of immune cells, such as neutrophils. Early recruitment of $\mathrm{NK}$ and cytotoxic $\mathrm{CD}^{+} \mathrm{T}$ cells is vital to the host to control virus infection as well as lung inflammation ${ }^{46,47}$. Other reported immunomodulatory activities of extracellular ISG15 include the antitumor activities of DCs ${ }^{48}$ and triggering the type II IFN response in NK and $T$ cells that is essential for immunity against mycobacteria ${ }^{49}$. Further characterization of the separable biochemical functions in animal models are called for to assess the specific core functions of ISG15 in response to infections. Delineating the roles of the free and conjugated forms of ISG15 should provide a better understanding of the pro-inflammatory and antiviral impact of ISG15 in virus pathogenesis and ascertain whether aberrant ISG15-dependent macrophage effector responses are universal features that underpin hyperinflammatory responses during infections by highly pathogenic viruses.

\section{Online content}

Any methods, additional references, Nature Research reporting summaries, source data, extended data, supplementary information, acknowledgements, peer review information; details of author contributions and competing interests; and statements of data and code availability are available at https://doi.org/10.1038/ s41590-021-01035-8.

Received: 22 August 2020; Accepted: 25 August 2021; Published online: 18 October 2021

\section{References}

1. McNab, F., Mayer-Barber, K., Sher, A., Wack, A. \& O'Garra, A. Type I interferons in infectious disease. Nat. Rev. Immunol. 15, 87-103 (2015).

2. García-Sastre, A. Induction and evasion of type I interferon responses by influenza viruses. Virus Res. 162, 12-18 (2011).

3. Taubenberger, J. K. \& Morens, D. M. The pathology of influenza virus infections. Annu. Rev. Pathol. 3, 499-522 (2008).

4. Channappanavar, R. \& Perlman, S. Pathogenic human coronavirus infections: causes and consequences of cytokine storm and immunopathology. Semin. Immunopathol. 39, 529-539 (2017).

5. Davidson, S., Crotta, S., McCabe, T. M. \& Wack, A. Pathogenic potential of interferon $\alpha \beta$ in acute influenza infection. Nat. Commun. 5, 3864 (2014).

6. Channappanavar, R. et al. Dysregulated type I interferon and inflammatory monocyte-macrophage responses cause lethal pneumonia in SARS-CoV-infected mice. Cell Host Microbe 19, 181-193 (2016).

7. Cheung, C. Y. et al. Induction of proinflammatory cytokines in human macrophages by influenza A (H5N1) viruses: a mechanism for the unusual severity of human disease? Lancet 360, 1831-1837 (2002).

8. Zhou, J. et al. Differential expression of chemokines and their receptors in adult and neonatal macrophages infected with human or avian influenza viruses. J. Infect. Dis. 194, 61-70 (2006).

9. Oslund, K. L. \& Baumgarth, N. Influenza-induced innate immunity: regulators of viral replication, respiratory tract pathology \& adaptive immunity. Future Virol. 6, 951-962 (2011).

10. McGonagle, D., Sharif, K., O’Regan, A. \& Bridgewood, C. The role of cytokines including interleukin-6 in COVID-19 induced pneumonia and macrophage activation syndrome-like disease. Autoimmun. Rev. 19, 102537 (2020).

11. Short, K. R., Kroeze, E. J. B. V., Fouchier, R. A. M. \& Kuiken, T. Pathogenesis of influenza-induced acute respiratory distress syndrome. Lancet Infect. Dis. 14, 57-69 (2014). 
12. Jahan, A. S. et al. OTUB1 is a key regulator of RIG-I-dependent immune signaling and is targeted for proteasomal degradation by influenza A NS1. Cell Rep. 30, 1570-1584.e6 (2020).

13. Giannakopoulos, N. V. et al. Proteomic identification of proteins conjugated to ISG15 in mouse and human cells. Biochem. Biophys. Res. Commun. 336, 496-506 (2005).

14. Wong, J. J. Y., Pung, Y. F., Sze, N. S.-K. \& Chin, K.-C. HERC5 is an IFN-induced HECT-type E3 protein ligase that mediates type I IFN-induced ISGylation of protein targets. Proc. Natl Acad. Sci. USA 103, 10735-10740 (2006).

15. Sanyal, S. et al. Type I interferon imposes a TSG101/ISG15 checkpoint at the Golgi for glycoprotein trafficking during influenza virus infection. Cell Host Microbe 14, 510-521 (2013).

16. Morales, D. J. et al. Novel mode of ISG15-mediated protection against influenza A virus and Sendai virus in mice. J. Virol. 89, 337-349 (2015).

17. Zhao, C., Hsiang, T.-Y., Kuo, R.-L. \& Krug, R. M. ISG15 conjugation system targets the viral NS1 protein in influenza A virus-infected cells. Proc. Nat Acad. Sci. USA 107, 2253-2258 (2010).

18. Farrell, P. J., Broeze, R. J. \& Lengyel, P. Accumulation of an mRNA and protein in interferon-treated Ehrlich ascites tumour cells. Nature 279, 523-525 (1979).

19. Haas, A. L., Ahrens, P., Bright, P. M. \& Ankel, H. Interferon induces a 15-kilodalton protein exhibiting marked homology to ubiquitin. J. Biol. Chem. 262, 11315-11323 (1987).

20. Hsiang, T.-Y., Zhao, C. \& Krug, R. M. Interferon-induced ISG15 conjugation inhibits influenza A virus gene expression and replication in human cells. J. Virol. 83, 5971-5977 (2009).

21. Kroeker, A. L., Ezzati, P., Halayko, A. J. \& Coombs, K. M. Response of primary human airway epithelial cells to influenza infection: a quantitative proteomic study. J. Proteome Res. 11, 4132-4146 (2012).

22. Radoshevich, L. ISG15 counteracts Listeria monocytogenes infection. eLife 4 , e06848 (2015)

23. Park, J. H. et al. Positive feedback regulation of p53 transactivity by DNA damage-induced ISG15 modification. Nat. Commun. 7, 12513 (2016).

24. Dos Santos, P. F. \& Mansur, D. S. Beyond ISGlylation: functions of free intracellular and extracellular ISG15. J. Interferon Cytokine Res. 37, 246-253 (2017).

25. Hermann, M. \& Bogunovic, D. ISG15: in sickness and in health. Trends Immunol. 38, 79-93 (2017)

26. Swaim, C. D., Scott, A. F., Canadeo, L. A. \& Huibregtse, J. M. Extracellular ISG15 signals cytokine secretion through the LFA-1 integrin receptor. Mol. Cell 68, 581-590.e5 (2017).

27. Perng, Y.-C. \& Lenschow, D. J. ISG15 in antiviral immunity and beyond. Nat. Rev. Microbiol. 16, 423-439 (2018).

28. Zhang, M. et al. A translocation pathway for vesicle-mediated unconventional protein secretion. Cell 181, 637-652.e15 (2020).

29. Leidal, A. M. et al. The LC3-conjugation machinery specifies the loading of RNA-binding proteins into extracellular vesicles. Nat. Cell Biol. 22, 187-199 (2020).

30. Ponpuak, M. et al. Secretory autophagy. Curr. Opin. Cell Biol. 35, 106-116 (2015).
31. Blott, E. J. \& Griffiths, G. M. Secretory lysosomes. Nat. Rev. Mol. Cell Biol. 3, 122-131 (2002)

32. Li, M. Y. et al. Lyn kinase regulates egress of flaviviruses in autophagosome-derived organelles. Nat. Commun. 11, 5189 (2020).

33. Chen, G. et al. Clinical and immunological features of severe and moderate coronavirus disease 2019. J. Clin. Invest. 130, 2620-2629 (2020).

34. Tay, M. Z., Poh, C. M., Rénia, L., MacAry, P. A. \& Ng, L. F. P. The trinity of COVID-19: immunity, inflammation and intervention. Nat. Rev. Immunol. 20, 363-374 (2020).

35. Koutsakos, M. et al. Downregulation of MHC class I expression by influenza A and B viruses. Front. Immunol. 10, 1158 (2019).

36. Pinto-Fernandez, A. et al. Deletion of the deISGylating enzyme USP18 enhances tumour cell antigenicity and radiosensitivity. Br. J. Cancer 124, 817-830 (2021)

37. Zhang, Y. et al. The in vivo ISGylome links ISG15 to metabolic pathways and autophagy upon Listeria monocytogenes infection. Nat. Commun. 10, 5383 (2019).

38. Shin, D. et al. Papain-like protease regulates SARS-CoV-2 viral spread and innate immunity. Nature 587, 657-662 (2020).

39. Zhang, $X$. et al. Human intracellular ISG15 prevents interferon- $\alpha / \beta$ over-amplification and auto-inflammation. Nature 517, 89-93 (2015).

40. Yan, S. et al. IRF3 reduces adipose thermogenesis via ISG15-mediated reprogramming of glycolysis. J. Clin. Invest. 131, e144888 (2021)

41. Tate, M. D., Pickett, D. L., van Rooijen, N., Brooks, A. G. \& Reading, P. C. Critical role of airway macrophages in modulating disease severity during influenza virus infection of mice. J. Virol. 84, 7569-7580 (2010).

42. Yu, W. C. L. et al. Viral replication and innate host responses in primary human alveolar epithelial cells and alveolar macrophages infected with influenza H5N1 and H1N1 viruses. J. Virol. 85, 6844-6855 (2011).

43. Wang, J. et al. Innate immune response of human alveolar macrophages during influenza A infection. PLoS ONE 7, e29879 (2012).

44. Swaim, C. D. et al. Modulation of extracellular ISG15 signaling by pathogens and viral effector proteins. Cell Rep. 31, 107772 (2020).

45. Mah, A. Y. et al. Glycolytic requirement for NK cell cytotoxicity and cytomegalovirus control. JCI Insight 2, e95128 (2017).

46. Wen, W. et al. Immune cell profiling of COVID-19 patients in the recovery stage by single-cell sequencing. Cell Discov. 6, 31 (2020).

47. Wang, Z. et al. Recovery from severe H7N9 disease is associated with diverse response mechanisms dominated by $\mathrm{CD}^{+} \mathrm{T}$ cells. Nat. Commun. 6 , 6833 (2015).

48. Padovan, E. et al. Interferon stimulated gene 15 constitutively produced by melanoma cells induces e-cadherin expression on human dendritic cells. Cancer Res. 62, 3453-3458 (2002).

49. Bogunovic, D. et al. Mycobacterial disease and impaired IFN- $\gamma$ immunity in humans with inherited ISG15 deficiency. Science 337, 1684-1688 (2012).

Publisher's note Springer Nature remains neutral with regard to jurisdictional claims in published maps and institutional affiliations.

(C) The Author(s), under exclusive licence to Springer Nature America, Inc. 2021 


\section{Methods}

Plasmid construction. The PLpro domain sequence was obtained from the SARS-CoV-2 complete genome (National Center for Biotechnology Information Genome databank: NC_045512.2). The protein sequence for the PLpro domain (amino acids 746-1,059) of pDONR207 SARS-CoV-2 Nsp3 (catalog no. 141257; Addgene) was cloned into a pCAGGs vector with a C-terminal FLAG-tag. The catalytic mutant $(\mathrm{C} 111 \mathrm{~A})$ was generated by site mutation PCR and verified with sequencing. The plasmid expressing PLpro was cloned in the pET28 expression vector using NcoI and XhoI restriction enzymes (provided by I. Ahel) for bacterial expression. The catalytic dead mutant of PLpro C111A was made using the Q5 Site-Directed Mutagenesis Kit (New England Biolabs).

Virus cultures. Influenza virus gene segments were amplified by PCR using Pfu Turbo DNA polymerase (catalog no. 600250; Stratagene) and cloned into the pHW2000 vector (a gift from R. G. Webster). Individual plasmids containing the eight viral genome segments were cotransfected using TransIT-LT1 Transfection Reagent (catalog no. MIR2300; Mirus Bio) into 293T (catalog no. CRL-3216, ATCC; research resource identifier (RRID): CVCL_0063) and MDCK cocultures (catalog no. CCL-34, ATCC; RRID: CVCL_0422). Recombinant viruses generated from the transfection system were propagated in embryonated eggs and quantified by plaque assay. Zika (strain MR766) and SARS-CoV-2 (Wu/01) virus stocks were prepared by determining the tissue culture infective dose $50 \%(\mathrm{ml})$ in Vero E6 cells challenged with tenfold serial dilutions of infectious supernatants for $90 \mathrm{~min}$ at $37^{\circ} \mathrm{C}$. Cells were subsequently incubated in DMEM with $2.5 \%$ FCS.

Cells and cell Lines. HeLa ISG15 $15^{-/-}$cells have been described previously ${ }^{50}$.

Primary cell culture and preparation for infection. For the influenza infections, buffy coat packs from healthy donors were kindly provided by the Hong Kong Red Cross Blood Transfusion Service; autologous plasma was collected after centrifugation from the top layer. The study received ethical approval from the institutional review board of the Li Ka Shing (LKS) Faculty of Medicine of the University of Hong Kong (no. UW 17-050). The remaining portion was mixed with Roswell Park Memorial Institute (RPMI) 1640 medium (catalog no. 23400021; Gibco), overlaid onto Ficoll-Paque PLUS density medium (catalog no. 17144003; GE Healthcare Life Sciences) and centrifuged at $1,000 \mathrm{~g}$ for $20 \mathrm{~min}$. Peripheral blood mononuclear cells were collected from the medium-Ficoll interface. Plastic-adherent monocytes were cultured in 5\% heat-inactivated autologous plasma in RPMI 1640 medium and allowed to differentiate for $14 \mathrm{~d}$ into macrophages. For DC differentiation, $50 \mathrm{ng} \mathrm{ml}^{-1}$ granulocyte-macrophage colony-stimulating factor (catalog no. 300-03; PeproTech), $10 \mathrm{ng} \mathrm{ml}^{-1}$ IL-4 (catalog no. 200-04; PeproTech) and $5 \%$ heat-inactivated autologous plasma in RPMI 1640 medium were used. NHBE cells were cultured in BEGM BulletKit Growth Medium (catalog no. CC-3170; Lonza). The day before influenza virus infection, cells were collected in trypsin/ EDTA; $0.1 \times 10^{6}$ cells were seeded in 24 -well cell culture plates (TPP).

Induced pluripotent stem cell-derived macrophages. For Zika and SARS-CoV-2 infections, induced pluripotent stem cell (iPSC)-derived CD14 ${ }^{+}$monocytes (ATCC) were resuspended in macrophage differentiation base medium (RPMI $1640,10 \%$ heat-inactivated FCS, $2 \mathrm{mM}$ of L-glutamine, $100 \mathrm{U} \mathrm{ml}^{-1}$ penicillin/ streptomycin) supplemented with $100 \mathrm{ng} \mathrm{ml}^{-1}$ of M-CSF. Cells were counted and seeded at a density of 150,000 precursor cells per well of a 6-well plate. Cells were cultured at $37^{\circ} \mathrm{C}$ for $6 \mathrm{~d}$ to differentiate into mature macrophages. At day 7 , cell density was verified to be $2-3$ times that of the initial number of precursors. Mature differentiated macrophages were infected with either Zika or SARS-CoV-2 for downstream functional assays.

Collection of plasma samples. Patients with RT-qPCR-confirmed COVID-19 disease at the Infectious Disease Centre of the Princess Margaret Hospital, Hong Kong, were invited to participate in the study after providing informed consent. The study was approved by the institutional review board of the Hong Kong West Cluster of the Hospital Authority of Hong Kong (approval no. UW20-169). Day 1 of clinical onset was defined as the first day of the appearance of clinical symptoms. Specimens of heparinized blood were collected from the patients and plasma was separated by centrifugation. Plasma was stored at $-80^{\circ} \mathrm{C}$ until use.

Virus infection. Cells were infected with the indicated strains of viruses at $37^{\circ} \mathrm{C}$ in the corresponding culture medium under serum-free condition for $1 \mathrm{~h}$. The virus inoculum was then removed, cells were washed with warm PBS and replenished with medium supplemented with $100 \mathrm{U} \mathrm{ml}^{-1}$ of penicillin, $100 \mu \mathrm{g} \mathrm{ml}^{-1}$ of penicillin-streptomycin (catalog no. 15140122; Gibco) and $1 \mu \mathrm{gl}^{-1}$ TPCK-treated trypsin (catalog no. T1426; Sigma-Aldrich) in case of influenza.

Gene silencing by RNA interference. All gene-specific DsiRNA oligonucleotides were purchased from Integrated DNA Technologies (Supplementary Table 4). DsiRNA was transfected with Viromer BLUE transfection reagent (catalog no. VB-01LB-01; Lipocalyx) at a final concentration of $25 \mathrm{nM}$. Forty-eight hours post-transfection, cells were either collected for analysis or subjected to additional experimental procedures as described.
ELISA. Cellular and secreted ISG15 were quantified with the human ISG15 ELISA kit (catalog no. CY-8085; MBL) according to the manufacturer's instructions. Briefly, $100 \mu \mathrm{l}$ of cleared cell lysate or culture medium were captured on ELISA assay plates coated with anti-ISG15. After extensive washing in buffer containing $0.2 \%$ Tween-20, $100 \mu \mathrm{l}$ of horseradish peroxidase anti-ISG15 was added for ISG15 protein detection. Binding was visualized by adding $100 \mu \mathrm{l}$ of $3,3^{\prime}, 5,5^{\prime}$-tetramethylbenzidine substrate and absorbance was measured at $450 \mathrm{~nm}$. For background correction, absorbance values at $550 \mathrm{~nm}$ were also measured and plotted against ISG15 protein standards. ISG15 protein concentration was then calculated using a linear regression method.

Cytokine quantification. Pro-inflammatory cytokine and chemokine concentrations were measured using a cytometric beads assay kit (catalog no. 740003; BioLegend), as advised by the manufacturer. Briefly, cytokine/chemokine standards or cleared culture supernatants were mixed with capture beads together with a biotin-conjugated antibody cocktail for $2 \mathrm{~h}$. Phycoerythrin-conjugated streptavidin was then added to the mixture and incubated for $30 \mathrm{~min}$. Beads were pelleted by centrifugation at $3,000 \mathrm{~g}$ for $5 \mathrm{~min}$, washed, fixed in $4 \%$ formaldehyde for $15 \mathrm{~min}$, centrifuged at $3,000 \mathrm{~g}$ for $5 \mathrm{~min}$ and resuspended in $250 \mu \mathrm{l}$ of $1 \mathrm{X}$ wash buffer for flow cytometry (LSRFortessa; BD) acquisition. Results were analyzed with the FCAP array software v.3.0 (BD). Specific capture beads groups were first separated by forward and side scatter dot plot graphs and further gated by allophycocyanin fluorescence channel. Phycoerythrin fluorescence readouts were then curve-fitted to a standard curve to estimate the concentration of analytes.

Phagocytosis assay. Macrophages $\left(1 \times 10^{6}\right)$ were seeded in $35-\mathrm{mm}$ non-cell culture-treated polystyrene dish in $500 \mu \mathrm{l}$ of RPMI 1640 supplemented with $5 \%$ autologous plasma, $100 \mathrm{U} \mathrm{ml}^{-1}$ of penicillin and $100 \mu \mathrm{g} \mathrm{ml}^{-1}$ of streptomycin. Macrophages were infected with the specified strains of influenza virus (MOI=2) in fresh serum-free medium (catalog no. 12065074; Gibco). At $24 \mathrm{~h}$ post-infection, $1 \times 10^{7}$ blue, fluorescent latex beads ( $1 \mu \mathrm{m}, 10$ beads per cell; Spherotech) were added and incubation continued for $1 \mathrm{~h}$ at $37^{\circ} \mathrm{C}$. Cells were then detached in $10 \mathrm{mM}$ of EDTA at $4^{\circ} \mathrm{C}$ for $20 \mathrm{~min}$, centrifuged at $250 \mathrm{~g}$ for $5 \mathrm{~min}$ at $4^{\circ} \mathrm{C}$ and eventually fixed in $4 \%$ formaldehyde at room temperature for $15 \mathrm{~min}$. Macrophages were pelleted at $500 \mathrm{~g}$ for $5 \mathrm{~min}$ and resuspended in $250 \mu \mathrm{l}$ of PBS, pH 7.4, for flow cytometry acquisition as described above.

Drug treatment. Macrophages $\left(2 \times 10^{5}\right)$ were seeded in 24 -well plates in $500 \mu \mathrm{l}$ of RPMI 1640 supplemented with $5 \%$ autologous plasma, $100 \mathrm{U} \mathrm{ml}^{-1}$ of penicillin and $100 \mu \mathrm{g} \mathrm{ml}^{-1}$ of streptomycin. Twenty-four hours later, the medium was discarded and replaced with fresh serum-free medium, which was changed daily for $2 \mathrm{~d}$. On the day of the experiment, macrophages were pretreated or post-treated with the indicated drug concentrations as detailed.

Protein expression and purification. pET28 WT and mutant PLpro plasmids were transformed into Rosetta DE3 expression cells and grown in lysogeny broth medium supplemented with kanamycin and chloramphenicol. Culture was induced with $0.5 \mathrm{mM}$ of isopropylthio- $\beta$-galactoside and $1 \mathrm{mM}$ of zinc chloride at $0.5-0.6 \mathrm{OD}_{600}$ and grown overnight at $16^{\circ} \mathrm{C}$. Protein purification was performed as described elsewhere ${ }^{38}$. The bacterial pellet was suspended in lysis buffer $(50 \mathrm{mM}$ of Tris- $\mathrm{HCl}, \mathrm{pH} 8,150 \mathrm{mM}$ of $\mathrm{NaCl}, 10 \mathrm{mM}$ of imidazole and $2 \mathrm{mM}$ of dithiothreitol (DTT)) and lysed using an homogenizer. Cleared lysate was incubated with prewashed Ni-NTA agarose resin (50\% slurry; QIAGEN) at $4{ }^{\circ} \mathrm{C}$ for $1 \mathrm{~h}$. Beads were further washed with increasing imidazole concentrations $(50 \mathrm{mM}$ of Tris- $\mathrm{HCl}, \mathrm{pH}$ $8,150 \mathrm{mM}$ of $\mathrm{NaCl}, 10-40 \mathrm{mM}$ of imidazole and $2 \mathrm{mM}$ of DTT). Histidine-tagged PLpro protein was eluted in elution buffer $(50 \mathrm{mM}$ of Tris- $\mathrm{HCl}, \mathrm{pH} 8,150 \mathrm{mM}$ of $\mathrm{NaCl}, 250 \mathrm{mM}$ of imidazole and $2 \mathrm{mM}$ of DTT). Eluted protein was dialyzed overnight in dialysis buffer $(20 \mathrm{mM}$ of Tris, $\mathrm{pH} 8,100 \mathrm{mM}$ of $\mathrm{NaCl}$ and $2 \mathrm{mM}$ of DTT) and further purified by size-exclusion chromatography using a Superdex75 column equilibrated with $20 \mathrm{mM}$ of Tris, $\mathrm{pH} 8,100 \mathrm{mM}$ of $\mathrm{NaCl}$ and $1 \mathrm{mM}$ of DTT.

Activity-based assay for PLpro. HA-tagged Ub-PA and ISG15-PA were used to perform activity-based probe assay with PLpro proteins. Briefly, activity probes (Ub-PA and ISG15-PA) were diluted in probe buffer $(50 \mathrm{mM}$ of Tris- $\mathrm{HCl}, \mathrm{pH} 7.5$, $150 \mathrm{mM}$ of $\mathrm{NaCl}$ ) and used at a $10-\mu \mathrm{M}$ final concentration in each reaction. WT and mutant PLpro proteins were used at different concentrations as indicated in the figures and prepared in activation buffer $(25 \mathrm{mM}$ of Tris- $\mathrm{HCl}, \mathrm{pH} 7.5$, $150 \mathrm{mM}$ of $\mathrm{NaCl}, 10 \mathrm{mM}$ of DTT). The reaction was initiated by mixing equal volumes of activated protein and activity probe and incubating the reaction at room temperature for $30 \mathrm{~min}$; it was stopped by adding SDS loading dye. Samples were run on a $4-12 \%$ Bis-Tris protein gel (Invitrogen) and visualized by Coomassie staining.

Secretome identification. LC-MS analyses for secretome identification were performed using a Thermo Fisher Scientific Orbitrap Lumos instrument that was operated in a data-dependent acquisition mode to switch between Orbitrap full-scan MS and LTQ MS/MS. Mass spectra were analyzed by MaxQuant v.1.4.1.2 and the Andromeda search engine. The maximum mass deviation allowed for the monoisotopic precursor ions was 4.5 parts per million ( $\mathrm{ppm}$ ) for monoisotopic 
precursors and $0.5 \mathrm{Da}$ for fragment ions. Trypsin was set as the digestion enzyme with a maximum of two allowed missed cleavages. Cysteine carbamidomethylation was set as a fixed modification and $\mathrm{N}$-terminal acetylation and methionine oxidation were allowed as variable modifications. Spectra were searched using the Andromeda search engine against the human UniProt (https://www.uniprot.org) sequence database. Protein identification required at least two unique peptides per protein group. Data were filtered for a $1 \%$ false discovery rate (FDR) at the peptide and protein level. PCA was performed using the Perseus software v.1.6.2.1 on the proteins common between the cells expressing NT DsiRNA or those targeting ISG15 or UBE1L. Protein expression fold change values were log-transformed (base 2); log-transformed fold change data showed bell shaped distributions and were symmetric around 0 .

Sample preparation for PLpro ISGylome. HeLa WT and Isg15 ${ }^{-/-}$cells were cultured in DMEM (catalog no. 31966047; Thermo Fisher Scientific) supplemented with 10\% FCS (catalog no. 10270106; Thermo Fisher Scientific) and maintained in a humidified incubator at $37^{\circ} \mathrm{C}$ and $5 \% \mathrm{CO}_{2}$. Five million cells of each genotype were seeded in triplicate for each condition (WT or mutant PLpro) in $12 \times 150 \mathrm{~mm}^{2}$ culture dishes (Greiner Bio-One). Cells were stimulated for $72 \mathrm{~h}$ with $500 \mathrm{U} \mathrm{ml}^{-1}$ IFN- $\alpha$ (catalog no. 11343504; ImmunoTools), washed in PBS and lysed in $50 \mathrm{mM}$ of Tris- $\mathrm{HCl}, \mathrm{pH} 8,150 \mathrm{mM}$ of $\mathrm{NaCl}, 1 \%$ Triton X-100, $1 \mathrm{mM}$ of phenylmethylsulfonyl fluoride (Sigma-Aldrich) and $1 \times$ protease inhibitor cocktail (Roche). Crude lysates were sonicated by 3 bursts of $10 \mathrm{~s}$ at $5 \mathrm{~W}$ output followed by centrifugation at $16,000 \mathrm{~g}$ for $15 \mathrm{~min}$ at $4{ }^{\circ} \mathrm{C}$. The protein content of the cleared lysates was measured by Bradford protein assay and $7.2 \mathrm{mg}$ of total protein of each replicate was treated with WT or mutant PLpro at a 1:50 (w/w) ratio for $30 \mathrm{~min}$ at $37^{\circ} \mathrm{C}$. To quench the activity of PLpro, $5 \%$ SDS was added to each replicate followed by reduction with DTT ( $4.5 \mathrm{mM})$ for $30 \mathrm{~min}$ at $55^{\circ} \mathrm{C}$ and alkylation with chloroacetamide $(10 \mathrm{mM})$ for $15 \mathrm{~min}$ at room temperature in the dark. Samples were subsequently prepared for MS analysis using the PTMScan HS Ubiquitin/SUMO Remnant Motif (K- $\varepsilon-\mathrm{GG}$ ) Kit (catalog no. 59322; Cell Signaling Technology). Briefly, all proteins were immobilized on S-Trap midi columns (C02-mini-40; ProtiFi) and digested on column with 1:100 (w/w) trypsin (catalog no. V5111; Promega Corporation) at $37^{\circ} \mathrm{C}$ overnight. The resulting peptides were eluted from the column, dried in a vacuum concentrator and redissolved in $1 \times$ immunoprecipitation buffer before immunocapture of GG-modified peptides. At this point, an aliquot of $30 \mu \mathrm{g}$ total peptide was taken for shotgun proteomics analysis. The remaining peptide solution was incubated with antibody bead slurry for $2 \mathrm{~h}$ at $4{ }^{\circ} \mathrm{C}$. Beads were washed and GG-modified peptides were eluted by adding $0.15 \%$ trifluoroacetic acid (TFA). Captured peptides were desalted on reversed-phase C18 OMIX tips (Agilent Technologies), dried under vacuum in high-performance LC inserts and stored at $-20^{\circ} \mathrm{C}$ until further use.

LC-MS/MS and data analysis for ISGylome determination. Dried GG-modified peptides were redissolved in $33 \mu \mathrm{l}$ of loading solvent A $(0.1 \%$ TFA in water/ acetonitrile $(98: 2, \mathrm{v} / \mathrm{v}))$ and $15 \mu \mathrm{l}$ was injected for LC-MS/MS analysis on an Ultimate 3000 RSLCnano system connected in line to a Q Exactive HF mass spectrometer (Thermo Fisher Scientific). Trapping was performed at $10 \mu \mathrm{lmin}^{-1}$ for $4 \mathrm{~min}$ in loading solvent $\mathrm{A}$ on a 20-mm trapping column (made in house, $100-\mu \mathrm{m}$ internal diameter, 5 - $\mu \mathrm{m}$ beads, C18 Reprosil-HD; Dr. Maisch) and the sample was loaded on a $250-\mathrm{mm}$ NanoEase analytical column $(75-\mu \mathrm{m}$ internal diameter, $1.8-\mu \mathrm{m}$ beads, C18 HSS T3; Waters). Peptides were eluted by a nonlinear increase from 1 to $55 \%$ MS solvent B $(0.1 \%$ formic acid in water/ acetonitrile $(2: 8, \mathrm{v} / \mathrm{v}))$ over $135 \mathrm{~min}$ at a constant flow rate of $300 \mathrm{nl} \mathrm{min}{ }^{-1}$, followed by a 15 -min wash reaching $97 \%$ MS solvent B and re-equilibration with MS solvent A ( $0.1 \%$ formic acid in water/acetonitrile $(98: 2, \mathrm{v} / \mathrm{v}))$. The column temperature was kept constant at $40^{\circ} \mathrm{C}$ in a column oven (CoControl 3.3.05; Sonation). The mass spectrometer was operated in data-dependent mode, automatically switching between MS and MS/MS acquisition for the eight most abundant ion peaks per MS spectrum. Full-scan MS spectra $(375-1,500 \mathrm{~m} / z)$ were acquired at a resolution of 60,000 in the Orbitrap analyzer after accumulation to a target value of $3,000,000$. The 8 most intense ions above a threshold value of 8,300 were isolated for fragmentation at a normalized collision energy of $28 \%$ after filling the trap at a target value of 100,000 for a maximum of $120 \mathrm{~ms}$. MS/MS spectra $(200-2,000 \mathrm{~m} / z)$ were acquired at a resolution of 15,000 in the Orbitrap analyzer. From the aliquots for the shotgun proteomics analysis, approximately $2 \mu \mathrm{g}$ of peptides were injected on the same LC-MS/MS system using similar settings as described above. The 16 most intense ions above a threshold value of 13,000 were isolated for fragmentation after filling the trap at a target value of 100,000 for a maximum of $80 \mathrm{~ms}$.

All data were analyzed in MaxQuant v.1.6.17.0 using the Andromeda search engine with default search settings, including an FDR set at $1 \%$ at the peptide and protein level. Separate searches were performed for the GG-peptidomics and shotgun samples. In both cases, spectra were searched against the human protein sequences in the UniProt database (database release version: January 2021 ) containing 20,621 sequences. Mass tolerance was set at $4.5 \mathrm{ppm}$ for precursor ions and $20 \mathrm{ppm}$ or $0.5 \mathrm{Da}$ for fragment ions of the GG-peptidomics and shotgun samples, respectively. Digestion mode was set to 'trypsin allow
P' enzyme specificity with up to three missed cleavages. Both searches included carbamidomethylation of cysteine residues as a fixed modification and oxidation of methionine and acetylation of protein $\mathrm{N}$ termini as variable modifications. In addition, GG-modification of lysine residues was added as a variable modification for the GG-peptidomics samples. The minimum score for identification of modified peptides was set to 30 , which led to the discovery of 2,143 GG-modification sites (listed in the $\operatorname{GlyGly}(\mathrm{K})$ site table from MaxQuant). For the shotgun samples, only proteins with at least 1 unique or razor peptide were retained resulting in 3,130 identified human proteins (listed in the proteinGroups table from MaxQuant)

GG-peptidomics data analysis was continued in Perseus v.1.6.2.1 after uploading the GlyGly(K) site table from MaxQuant. The data were processed by removing reverse sequences, potential contaminants and sites with a localization probability less than 0.75 . Afterwards, the site table was expanded and the intensities were $\log _{2}$-transformed. The data was normalized through subtraction of the median intensity in each sample. Next, replicates were grouped and sites with fewer than three valid values in at least one replicate group were discarded. To allow statistical testing, missing data values were imputed from a normal distribution around the detection limit. The experimental design was uploaded into the site table by defining groups based on treatment (WT or mutant PLpro) and genotype (HeLa WT or ISG15\%). A two-way analysis of variance (ANOVA) was done to compare the site intensities between treatment and genotype. Three $P$ values were calculated for each site, including a $P$ value for the effect of treatment and genotype and an interaction $P$ value. Sites with $P<0.01$ for at least 1 of the 3 parameters were retained and used for unsupervised hierarchical clustering after $z$-score normalization. Significantly regulated sites were grouped into clusters and visualized in a heatmap in Fig. 6 with their intensity per replicate across the different experimental groups. The significantly regulated modification sites are listed in Supplementary Table 1.

In similar fashion, the shotgun proteomics data were further analyzed in Perseus after uploading the proteinGroups table from MaxQuant. Reverse sequences, potential contaminants and proteins only identified by site were removed. Label-free quantitation (LFQ) intensities were $\log _{2}$-transformed and the data were normalized by subtracting the median intensity in each sample. Replicates were grouped and proteins with fewer than three valid values in at least one replicate group were discarded. Missing data values were imputed from a normal distribution around the detection limit. To reveal proteins that were significantly regulated, samples were grouped in the same way as described above and a two-way ANOVA was performed to compare the LFQ intensities of the proteins in the treatment group with the genotype group. Proteins with $P<0.01$ for at least 1 of the 3 parameters were considered to be significantly regulated. The LFQ intensities of these proteins after nonsupervised hierarchical clustering are shown in a heatmap in Supplementary Fig. 7. The significantly regulated proteins are shown in Supplementary Table 2 . As only 2 major clusters were observed in the heatmap, a $t$-test was performed $(\mathrm{FDR}=0.05$ and $\mathrm{S} 0=1)$ to compare protein intensities between all WT and Isg15 ${ }^{-/-}$samples. Quantified proteins $(n=2,438)$ and the results of the $t$-test are listed in Supplementary Table 3 and shown in the volcano plot in Supplementary Fig. 7.

Statistical analysis. Results are shown as the mean \pm s.e.m. or the mean \pm s.d. as indicated of experiments performed in three or four independent biological replicates. The statistical differences between groups were determined by two-tailed Mann-Whitney $U$-test with a confidence limit for significance set at 0.05 or less.

Software and code. Fluorescence-activated cell sorting (FACS) data were acquired on an Attune NxT Flow Cytometer and BD LSRFortessa. LC-MS/MS data were acquired on an Ultimate 3000 RSLCnano system connected in line to a Q Exactive HF mass spectrometer. All FACS data were analyzed using FlowJo v.10 (FlowJo LLC). MS data were analyzed in MaxQuant v.1.6.17.0 using the Andromeda search engine. GG-peptidomics data analysis was continued in Perseus v.1.6.2.1. Ingenuity Pathway Analyses (March 2020 version), Panther v.16.0 and DAVID v.6.8 were used to perform gene ontology analyses and Prism v.8 (GraphPad Software) was used for the statistical analyses.

Reporting Summary. Further information on research design is available in the Nature Research Reporting Summary linked to this article.

\section{Data availability}

The protein sequences used in this study were extracted from spectra and were searched against the human protein sequences in the UniProt database (January 2021 release) containing 20,621 sequences (https://www.uniprot.org) (human, including isoforms and unreviewed sequences). Mass spectrometry data have been deposited with the ProteomeXchange Consortium through the PRIDE repository under accession no. PXD026748. All accession numbers of the RNA interference experiments have been provided in the supplementary information. Source files for the immunoblots presented in the manuscript have been provided. Data generated or analyzed during the current study are available through Figshare (https://figshare.com/s/e20b8ccale02219f16b9). Source data are provided with this paper. 


\section{References}

50. Kespohl, M. et al. Protein modification with ISG15 blocks coxsackievirus pathology by antiviral and metabolic reprogramming. Sci. Adv. 6, eaay1109 (2020).

\section{Acknowledgements}

We thank the Faculty Core Facility and Centre for Genomic Sciences of the LKS Faculty of Medicine of the University of Hoing Kong for providing tools and technical support; we also acknowledge the support of the clinicians who facilitated this study, including O. T.-Y. Tsang, W. S. Leung, J. M. C. Chan, T. S. H. Chik, C. Y. C. Choi, J. Y. H. Chan, D. P.-L. Lau and Y. M. Ho, the dedicated clinical team at the Infectious Diseases Centre, Princess Margaret Hospital, Hospital Authority of Hong Kong, the patients who kindly consented to participate in this investigation and the Hong Kong Red Cross Blood Transfusion Service for providing buffy coat packs for this research project. We thank R. Bruzzone for comments on the manuscript and J. S. M. Peiris for the license on the clinical experiments. This work was supported by research grants from the Research Grants Council of the Hong Kong Special Administrative Region (no. 17113019) (S.S.), Health and Medical Research Funds (no. 17161202) (S.S.), Medical Research Council UK (no. MC_PC_19063) (S.S.) and the Wellcome Trust (no. 220776/Z/20/Z) (S.S.), Guangdong Province International Scientific and Technological Cooperation Projects (no. 2020A0505100063) (C.K.P.M.) and the National Research Foundation of Korea grant funded through the Korean government (no. NRF-2018M3A9H4055203) (C.K.P.M.). F.I. acknowledges support from the Odysseus grant no. G0F8616N from Research Foundation Flanders and ERA-NET Infect-ERA BacVirISG15. D.E. is supported by a Research Foundation Flanders PhD fellowship for fundamental research.
A.B. receives support from the Foundation for Experimental Biomedicine Zurich, the Berlin University Alliance and the German Research Foundation (grant nos. BE 6335/2 and BE 6335/4 and CRC1292 project 02 and CRC167 Project-ID 259373024).

\section{Author contributions}

F.I., C.K.P.M. and S.S. designed and conducted the study. D.M., Q.T., D.E., H.H.Y.L., F.T., S.W.v.L., J.H., C.K.P.M., L.Y.L.S. and W.W.S.N. performed and analyzed the experiments. A.B., H.P., B.K., A.D. and A.P-F. generated the critical reagents for the study. S.S. and C.K.P.M. wrote the manuscript.

\section{Competing interests}

The authors declare no competing interests.

\section{Additional information}

Supplementary information The online version contains supplementary material available at https://doi.org/10.1038/s41590-021-01035-8.

Correspondence and requests for materials should be addressed to Francis Impens, Chris Ka Pun Mok or Sumana Sanyal.

Peer review information Nature Immunology thanks Xuetao Cao, Ivan Dikic and Trine $\mathrm{H}$. Mogensen for their contribution to the peer review of this work. Zoltan Fehervari was the primary editor on this article and managed its editorial process and peer review in collaboration with the rest of the editorial team. Peer reviewer reports are available.

Reprints and permissions information is available at www.nature.com/reprints. 


\section{Reporting Summary}

Nature Research wishes to improve the reproducibility of the work that we publish. This form provides structure for consistency and transparency in reporting. For further information on Nature Research policies, see Authors \& Referees and the Editorial Policy Checklist.

\section{Statistics}

For all statistical analyses, confirm that the following items are present in the figure legend, table legend, main text, or Methods section.

$\mathrm{n} / \mathrm{a}$ Confirmed

$\bigotimes$ The exact sample size $(n)$ for each experimental group/condition, given as a discrete number and unit of measurement

$\square$ \A statement on whether measurements were taken from distinct samples or whether the same sample was measured repeatedly

$\square$ The statistical test(s) used AND whether they are one- or two-sided

Only common tests should be described solely by name; describe more complex techniques in the Methods section.

$\square$ A description of all covariates tested

$\square$ \A description of any assumptions or corrections, such as tests of normality and adjustment for multiple comparisons

$\square$ A full description of the statistical parameters including central tendency (e.g. means) or other basic estimates (e.g. regression coefficient)

AND variation (e.g. standard deviation) or associated estimates of uncertainty (e.g. confidence intervals)

$\square$ For null hypothesis testing, the test statistic (e.g. $F, t, r$ ) with confidence intervals, effect sizes, degrees of freedom and $P$ value noted

Give $P$ values as exact values whenever suitable.

Х $\square$ For Bayesian analysis, information on the choice of priors and Markov chain Monte Carlo settings

Х $\square$ For hierarchical and complex designs, identification of the appropriate level for tests and full reporting of outcomes

$\square$ Estimates of effect sizes (e.g. Cohen's $d$, Pearson's $r$ ), indicating how they were calculated

Our web collection on statistics for biologists contains articles on many of the points above.

\section{Software and code}

Policy information about availability of computer code

Data collection FACS data was acquired on Attune NxT Flow Cytometer and BD LSRFortessa, LC-MS/MS data was acquired on an Ultimate 3000 RSLCnano system in-line connected to a Q Exactive HF mass spectrometer (Thermo Scientific).

Data analysis $\quad$ All FACS data were analysed using FlowJo 10. All MS data were analyzed in MaxQuant (version 1.6.17.0) using the Andromeda search engine. GG-peptidomics data analysis was continued in Perseus (version 1.6.2.1). Ingenuity Pathway Analyses (version March 2020), Panther (version 16.0) and DAVID (version 6.8) were used for performing gene ontology analyses and GraphPad Prism (version 8) for statistical analyses.

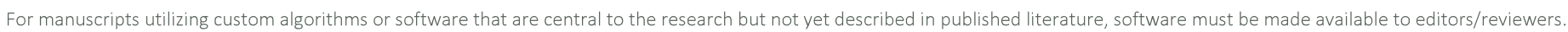
We strongly encourage code deposition in a community repository (e.g. GitHub). See the Nature Research guidelines for submitting code \& software for further information.

\section{Data}

Policy information about availability of data

All manuscripts must include a data availability statement. This statement should provide the following information, where applicable:

- Accession codes, unique identifiers, or web links for publicly available datasets

- A list of figures that have associated raw data

- A description of any restrictions on data availability

Protein sequences used in this study were extracted from UniprotKB (Human; release 2020_06 including isoforms and unreviewed sequences; https:// www.uniprot.org/uniprot//. Mass spectrometry data has been deposited to the ProteomeXchange Consortium through the PRIDE repository under the identifier PXD026748. All accession codes of RNAi experiments have been provided in the supplementary information. Source files for immunoblots presented in the manuscript have been provided. Data generated or analysed during the current study are available through Figshare. 
Please select the one below that is the best fit for your research. If you are not sure, read the appropriate sections before making your selection.
\ Life sciences
Behavioural \& social sciences
Ecological, evolutionary \& environmental sciences

For a reference copy of the document with all sections, see nature.com/documents/nr-reporting-summary-flat.pdf

\section{Life sciences study design}

All studies must disclose on these points even when the disclosure is negative.

Sample size Samples sizes were selected keeping in mind the variability between independent sources of cells. All experiments were performed in mammalian cell cultures, which are population-based, with data points generated from experiments performed from cells generated from independent clones and performed independent of each other. Sample sizes were determined based on the numbers required to achieve statistical significance using indicated statistics, but with a minimum of 3 independently performed experiments to ensure data reproducibility.

Data exclusions No data was excluded.

\begin{tabular}{l|l} 
Replication & For all experiments data were generated with three or four independent biological replicates along with atleast 2 technical replicates to
\end{tabular} calculate the mean and standard deviation. All attempts at replication were successful.

Randomization Cells from different clones were independently replicated on atleast 3 separate days, with wells in microtiter plates randomised for treatment conditions, and measurements taken on $\sim 1 \times$ E6 cells from each well.

Blinding Blinding was not performed in most experiments which required known treatments of cells with infectious pathogens. Therefore, it was not possible to blind the investigator for such experiments. Blinding was performed for wt and mutant PLpro in vitro assays.

\section{Reporting for specific materials, systems and methods}

We require information from authors about some types of materials, experimental systems and methods used in many studies. Here, indicate whether each material, system or method listed is relevant to your study. If you are not sure if a list item applies to your research, read the appropriate section before selecting a response.

\begin{tabular}{|c|c|c|c|}
\hline \multicolumn{2}{|r|}{ Materials \& experimental systems } & \multicolumn{2}{|c|}{ Methods } \\
\hline$n / a$ & Involved in the study & $\mathrm{n} / \mathrm{a}$ & Involved in the study \\
\hline$\square$ & $\bigotimes$ Antibodies & Х & $\square$ ChIP-seq \\
\hline $\mathbf{L}^{-}$ & Eukaryotic cell lines & $\square$ & \ Flow cytometry \\
\hline Х & $\square$ Palaeontology & Х & $\square$ MRI-based neuroimaging \\
\hline Х & $\square$ Animals and other organisms & & \\
\hline$\square$ & $\bigotimes$ Human research participants & & \\
\hline Х & $\square$ Clinical data & & \\
\hline
\end{tabular}

\section{Antibodies}

Antibodies used

mouse anti-ISG15 antibody (F-9 from Santa Cruz Biotechnology; 1:500), rabbit anti-ISG15 (Sigma Aldrich; Cat no: HPA004627); 1:500; rabbit anti-tubulin- $\alpha$ antibody (ab18251 from Abcam; 1:1,000) and mouse anti-ubiquitin antibody (P4D1 from Santa Cruz Biotechnology; sc-8017; 1:500), goat anti-mouse-IgG (IRDye ${ }^{\circledR}$ 800CW, Li-COR), goat anti-mouse-IgG (IRDye ${ }^{\circledR} 680 R D$, Li-COR) and goat anti-rabbit-IgG (IRDye ${ }^{\circledR}$ 680RD, Li-COR); rabbit anti-Lyn mAb (Cat no 2796, clone no C13F9, Cell Signaling), 1:1000; rabbit anti-SFK mAb (Cat no 2123, clone no 32G6, Cell Signaling Technology); 1:1000, mouse anti-GAPDH mAb - Anti-GAPDH antibody [6C5], (ab8245), 1:1500, mouse anti-MHC-I (clone W6/32), Abcam (ab224232), 1:1000, horseradish peroxidase (HRP)-conjugated secondary antibody (Jackson ImmunoResearch Cat\#115-035-003, RRID:AB_10015289, Anti-LAMP1 antibody (Cat no ab24170; 1:1000), Anti-Syntaxin 3 antibody [EPR8543] (ab133750); 1:500; Anti-Syntaxin 4 antibody [EPR15473] (ab184545); 1:1500, AntiSTX17 antibody (ab229646); 1:500, Anti-Tmed10 antibody [EPR9036(B)] (ab134948); 1:500, Anti-LC3B antibody [EPR18709] (ab192890); 1:1000

Validation

All antibody lots are routinely tested by the manufacturers, and each product comes with a certificate of analysis from the indicated vendor stating that the product has met all quality control standards. All antibodies that were purchased from commercial vendors which include this statement of certification. 
Policy information about cell lines

Cell line source(s)

Hela cells, Cat no ATCC ${ }^{\circledast}$, CCL-2 $^{\text {TM }}$, HeLa ISG15 KO cells (previously described; original source ATCC; Cat no ATCC ${ }^{\otimes}$, CCL-2 $^{\text {TM}}$ ), iPSC-derived CD14+ monocytes (from ATCC; DYS0100; Cat no ACS-7030), Vero E6 (ATCC; Cat no CRL-1586

Authentication

All cell lines were purchased from ATCC, which were characterized and authenticated by short tandem repeat (STR) DNA profiles.

Mycoplasma contamination

all cell lines tested and confirmed to be negative for mycoplasma

Commonly misidentified lines

(See ICLAC register)

No commonly misidentified cell lines (reported by ICLAC) have been used in this study.

\section{Human research participants}

Policy information about studies involving human research participants

Population characteristics

Describe the covariate-relevant population characteristics of the human research participants (e.g. age, gender, genotypic information, past and current diagnosis and treatment categories). If you filled out the behavioural \& social sciences study design questions and have nothing to add here, write "See above."

Recruitment Describe how participants were recruited. Outline any potential self-selection bias or other biases that may be present and how these are likely to impact results.

Ethics oversight

Identify the organization(s) that approved the study protocol.

Note that full information on the approval of the study protocol must also be provided in the manuscript.

\section{Flow Cytometry}

Plots

Confirm that:

\The axis labels state the marker and fluorochrome used (e.g. CD4-FITC).

Whe axis scales are clearly visible. Include numbers along axes only for bottom left plot of group (a 'group' is an analysis of identical markers).

\All plots are contour plots with outliers or pseudocolor plots.

\A numerical value for number of cells or percentage (with statistics) is provided.

\section{Methodology}

Sample preparation

Instrument

Software

Cell population abundance

Gating strategy

\Tick this box to confirm that a figure exemplifying the gating strategy is provided in the Supplementary Information.
At designated time points, the cells were dissociated with 10 mM EDTA in PBS at 37C. Cells were then stained with 1:200 Zombie NIR ${ }^{T M}$ Fixable Viability Kits or for 10 to 15 minutes. Unbound dyes were neutralized and washed away with FACS buffer (PBS containing $2 \% \mathrm{FBS}$ and $0.004 \%$ sodium azide [w/v]). Cells were washed with FACS buffer and fixed with $4 \%$ formaldehyde diluted in PBS. Cells were then permeabilized using BD Perm/Wash Buffer (BD Biosciecences; 554723) at room temperature for 20 minutes. Intracellular proteins were then stained with $50 \mu \mathrm{L}$ of indicated antibodies (e.g. W6/32 clone for MHC-I) diluted with BD Perm/Wash Buffer on ice for 30 minutes. Cells were then washed with BD Perm/Wash Buffer and resuspended in FACS buffer before data acquisition.

FACS data was acquired on Attune NxT Flow Cytometer.

All FACS data were analysed using FlowJo 10.

There is no sorting involved in this study.

For all the experiments, cells were first gated on single cells by a forward side scatter gate, followed by FITC gating by excluding dead cells. Populations were gated in consistence with the corresponding negative controls. 